\title{
Stability of a CTL-mediated immunity HIV infection models with silent infected cells and cellular infection
}

\author{
Noura H. AlShamrani ${ }^{\mathrm{a}, \mathrm{b}}$, Ahmed M. Elaiw ${ }^{\mathrm{b}, \mathrm{c}, *}$ \\ ${ }^{a}$ Department of Mathematics, Faculty of Science, University of Jeddah, P.O. Box 80327, Jeddah 21589, Saudi Arabia. \\ ${ }^{b}$ Department of Mathematics, Faculty of Science, King Abdulaziz University, P.O. Box 80203, Jeddah 21589, Saudi Arabia. \\ ${ }^{c}$ Department of Mathematics, Faculty of Science, Al-Azhar University, Assiut, Egypt.
}

\begin{abstract}
This paper proposes and analyzes a CTL-mediated HIV infection model. The susceptible CD4 ${ }^{+} \mathrm{T}$ cells can be infected when they are contacted by one of the following: (i) free HIV particles, (ii) silent infected cells, and (iii) actively infected cells. The effect of saturation infection has been incorporated in the second model. The model is an improvement of an existing HIV infection models which have neglected the infection due to incidence between the silently infected cells and susceptible CD4 ${ }^{+} \mathrm{T}$ cells. We first show that the models are well-posed. Each of our proposed models has three equilibria, namely: HIV-free equilibrium, $\mathrm{Ð}_{0}$, chronic HIV infection equilibrium with inactive CTL-mediated immune response, $\biguplus_{1}$, chronic HIV infection equilibrium with active CTL-mediated immune response, $\bigoplus_{2}$. We derive two threshold parameters, the basic HIV reproduction number, $\Re_{0}$, and the CTL-mediated immunity reproduction number, $\mathfrak{R}_{1}$. These parameters determine the existence and global stability of the equilibria of the model. We prove the global asymptotic stability of all equilibria by utilizing the Lyapunov function and LaSalle's invariance principle. We have proven the following: (i) if $\mathfrak{R}_{0} \leqslant 1$, then $\bigoplus_{0}$ is globally asymptotically stable (G.A.S), (ii) if $\mathfrak{R}_{1} \leqslant 1<\mathfrak{R}_{0}$, then $\bigoplus_{1}$ is G.A.S, and (iii) if $\mathfrak{R}_{1}>1$, then $\bigoplus_{2}$ is G.A.S. We have illustrated the theoretical results via numerical simulations. We have studied the effects of cell-to-cell (CTC) transmission and saturation on the dynamical behaviour of the system. We have shown that inclusion of CTC transmission decreases the concentration of susceptible CD4 ${ }^{+} \mathrm{T}$ cells and increases the concentrations of infected cells and free HIV particles. While the inclusion of saturation increases the concentration of susceptible CD4 ${ }^{+} \mathrm{T}$ cells and reduces the concentrations of infected cells and free HIV particles.
\end{abstract}

Keywords: HIV infection, viral and cellular infections, global stability, silent infected cells, CTL-mediated immune response, Lyapunov function.

2020 MSC: 34D20, 34D23, 37N25, 92B05.

(C)2021 All rights reserved.

\section{Introduction}

Acquired immunodeficiency syndrome (AIDS) is one of the dangerous human diseases which is caused by human immunodeficiency virus (HIV). The main target of the HIV is the susceptible CD4 ${ }^{+} \mathrm{T}$ cells which play an important role in the immune system. Cytotoxic T lymphocyte (CTL) and antibody immune responses can control the HIV infection for long period up to 10 years [5]. However, during this period of time the concentration of susceptible $\mathrm{CD} 4^{+} \mathrm{T}$ cells is declined. When the concentration of the $\mathrm{CD} 4{ }^{+} \mathrm{T}$ cells reaches below 200 cells $/ \mathrm{mm}^{3}$, the patient is said to have progressed to AIDS. During the last decades, mathematical modeling of a within-host HIV infection has witnessed a significant development. Stability analysis has also become one of the most important and fundamental approaches for

\footnotetext{
${ }^{*}$ Corresponding author

Email addresses: nhalshamrani@uj.edu.sa (Noura H. AlShamrani), a_m_elaiw@yahoo.com (Ahmed M. Elaiw)
}

doi: $10.22436 /$ jmcs.022.03.03

Received: 2020-05-16 Revised: 2020-07-03 Accepted: 2020-07-21 
understanding the within-host HIV dynamics. The basic mathematical model of a within-host HIV infection with CTL-mediated immunity has been introduced in [34]. The model contains four compartments, susceptible $\mathrm{CD}^{+}$T cells $(\mathrm{W})$, active HIV-infected cells $(\mathrm{M})$, free HIV particles $(\mathrm{N})$, and HIV-specific CTLs $(\mathrm{P})$.

$$
\left\{\begin{array}{l}
\dot{W}(t)=\rho-\alpha W(t)-\eta_{1} W(t) N(t) \\
\dot{M}(t)=\eta_{1} W(t) N(t)-a M(t)-\mu P(t) M(t) \\
\dot{N}(t)=b M(t)-\varepsilon N(t) \\
\dot{P}(t)=\sigma P(t) M(t)-\pi P(t)
\end{array}\right.
$$

The susceptible $\mathrm{CD} 4^{+} \mathrm{T}$ cells are produced at specific constant rate $\rho$ and die at rate $\alpha \mathrm{W}$. The term $\eta_{1} \mathrm{WN}$ refers to the rate at which new infectious appears by virus-cell contact between free HIV particles and susceptible $\mathrm{CD}^{+} \mathrm{T}$ cells. The active HIV-infected cells die at rate aM. The term $\mu \mathrm{PM}$ is the killing rate of active HIV-infected cells due to their HIV-specific CTL-mediated immunity. The free HIV particles are generated at rate $b M$ and cleared from the plasma at rate $\varepsilon N$. The proliferation rate of the effective HIV-specific CTLs is given by $\sigma P M$. The term $\pi$ P represents the decay rate of the CTLs. HIV infection models with CTL-mediated immune response have been investigated in many papers (see, e.g., [17, 18, $24,31,32,35,37,38,41,43,47])$. In model (1.1), it was assumed that the infection occurs due to viruscell contact known as virus-to-cell (VTC) transmission. Several works have reported that the susceptible $\mathrm{CD}^{+} \mathrm{T}$ cells can also be infected due to cell-cell contact known as cell-to-cell (CTC) transmission (see e.g. [25-27, 36]). Therefore, CTC transmission plays an important role in the HIV infection process even during the antiviral treatment [39]. The CTC transmission has been incorporated into viral infection models with $[6,14,15,21,42]$ or without $[1,8,16,19,29,30,46]$ CTL-mediated immune response.

It is known that highly active anti-retroviral therapy can suppress HIV replication to a low level but cannot enucleate the HIV from the body. One of the main reasons of this fact is the presence of silent (latent) $\mathrm{CD}^{+} \mathrm{T}$ infected cells where the HIV provirus can reside $[7,45]$. Silent infected cells live long, but it can be activated to produce new HIV particles. In a recent interesting discovery [2], it has been shown that both silent and active infected cells can infect the susceptible CD4 ${ }^{+} \mathrm{T}$ cells through $\mathrm{CTC}$ mechanism. In a very recent work, Wang et al. [44] have formulated a viral infection model by assuming that both silent and active infected cells can participate in CTC infection. However, in [44], the CTL immune response has not been considered.

In the present paper, we first formulate an HIV infection model with CTL-mediated immune response and both VTC and CTC transmissions. The CTC transmission is due to the contact of susceptible CD4 ${ }^{+} \mathrm{T}$ cells with silent or active HIV-infected cells. The effect of saturation infection has been incorporated into the second model. The well-posedness of each model is investigated by establishing that the solutions of the model are nonnegative and bounded. We derive two threshold parameters which determine the existence and stability of the three equilibria. Global stability of all equilibria is proven by formulating Lyapunov functions and utilizing LaSalle's invariance principle. We perform some numerical simulations to illustrate the strength of our theoretical results.

\section{Model formulation}

We formulate an HIV infection model by assuming that the HIV virions can replicate by two mechanisms, VTC and CTC transmissions. The CTC infection has two sources, (i) the contact between susceptible $\mathrm{CD} 4^{+} \mathrm{T}$ cells and silent HIV-infected cells, and (ii) the contact between susceptible $\mathrm{CD} 4^{+} \mathrm{T}$ cells and active HIV-infected cells. Under these assumptions we propose the following model:

$$
\left\{\begin{array}{l}
\dot{W}(t)=\rho-\alpha W(t)-\eta_{1} W(t) N(t)-\eta_{2} W(t) U(t)-\eta_{3} W(t) M(t), \\
\dot{U}(t)=\eta_{1} W(t) N(t)+\eta_{2} W(t) U(t)+\eta_{3} W(t) M(t)-\lambda U(t)-\gamma U(t), \\
\dot{M}(t)=\lambda U(t)-a M(t)-\mu P(t) M(t), \\
\dot{N}(t)=b M(t)-\varepsilon N(t), \\
\dot{P}(t)=\sigma P(t) M(t)-\pi P(t),
\end{array}\right.
$$


where $\mathrm{U}$ is the concentration of silent HIV-infected cells. The susceptible $\mathrm{CD} 4^{+} \mathrm{T}$ cells are contacted with silent HIV-infected cells and active HIV-infected cells and become infected due to CTC transmission at rates $\eta_{2} W U$ and $\eta_{3} W M$, respectively. The terms $\lambda U$ and $\gamma U$ are the rates of silent HIV-infected cells that become active and the natural death of the silent HIV-infected cells, respectively. All remaining variables and parameters have the same biological meaning as explained in Section 1. Table 1 summarizes all parameters and their definitions.

Table 1: Parameters of model (2.1) and their interpretations.

\begin{tabular}{ll}
\hline Parameter & Biological meaning \\
\hline$\rho$ & Recruitment rate for the susceptible CD4 ${ }^{+} \mathrm{T}$ cells \\
$\alpha$ & Natural death rate constant for the susceptible CD4 ${ }^{+} \mathrm{T}$ cells \\
$\eta_{1}$ & Virus-cell incidence rate constant between free HIV particles and susceptible CD4 ${ }^{+} \mathrm{T}_{\text {cells }}$ \\
$\eta_{2}$ & Cell-cell incidence rate constant between silent HIV-infected cells and susceptible CD4 ${ }^{+} \mathrm{T}$ cells \\
$\eta_{3}$ & Cell-cell incidence rate constant between active HIV-infected cells and susceptible CD4 ${ }^{+} \mathrm{T}_{\text {cells }}$ \\
$\gamma$ & Death rate constant of silent HIV-infected cells \\
$\mathrm{a}$ & Death rate constant of active HIV-infected cells \\
$\mu$ & Killing rate constant of active HIV-infected cells due to their specific CTL-mediated immunity \\
$\lambda$ & Transmission rate constant of silent HIV-infected cells that become active HIV-infected cells \\
$\mathrm{b}$ & Generation rate constant of new HIV particles \\
$\varepsilon$ & Death rate constant of free HIV particles \\
$\sigma$ & Proliferation rate constant of HIV-specific CTLs \\
$\pi$ & Decay rate constant of HIV-specific CTLs \\
\hline
\end{tabular}

\subsection{Well-posedness of solutions}

Let $\Omega_{j}>0, j=1,2,3$ and define the compact set

$$
Q=\left\{(W, U, M, N, P) \in \mathbb{R}_{\geqslant 0}^{5}: 0 \leqslant W(t), U(t), M(t) \leqslant \Omega_{1}, 0 \leqslant N(t) \leqslant \Omega_{2}, 0 \leqslant P(t) \leqslant \Omega_{3}\right\} .
$$

Proposition 2.1. The compact set $Q$ is positively invariant for system (2.1).

Proof. We have

$$
\begin{aligned}
& \left.\dot{W}\right|_{W=0}=\rho>0, \\
& \left.\dot{U}\right|_{U=0}=\eta_{1} W N+\eta_{3} W M \geqslant 0 \text { for all } W, N, M \geqslant 0, \\
& \left.\dot{M}\right|_{M=0}=\lambda U \geqslant 0 \text { for all } U \geqslant 0, \\
& \left.\dot{\mathrm{N}}\right|_{N=0}=b M \geqslant 0 \text { for all } M \geqslant 0, \\
& \left.\dot{P}\right|_{P=0}=0 .
\end{aligned}
$$

This ensures that $(W(t), U(t), M(t), N(t), P(t)) \in \mathbb{R}_{\geqslant 0}^{5}$ for all $t \geqslant 0$ when $(W(0), U(0), M(0), N(0), P(0)) \in$ $\mathbb{R}_{\geqslant 0}^{5}$. To show the boundedness of all state variables, we let

$$
\Psi(t)=W(t)+U(t)+M(t)+\frac{a}{2 b} N(t)+\frac{\mu}{\sigma} P(t) .
$$

Then

$$
\begin{aligned}
\dot{\Psi}(t) & =\rho-\alpha W(t)-\gamma U(t)-\frac{a}{2} M(t)-\frac{a \varepsilon}{2 b} N(t)-\frac{\mu \pi}{\sigma} P(t) \\
& \leqslant \rho-\phi\left(W(t)+U(t)+M(t)+\frac{a}{2 b} N(t)+\frac{\mu}{\sigma} P(t)\right)=\rho-\phi \Psi(t),
\end{aligned}
$$

where $\phi=\min \left\{\alpha, \gamma, \frac{a}{2}, \varepsilon, \pi\right\}$. It follows that,

$$
\Psi(\mathrm{t}) \leqslant e^{-\phi t}\left(\Psi(0)-\frac{\rho}{\phi}\right)+\frac{\rho}{\phi} .
$$

Hence, $0 \leqslant \Psi(t) \leqslant \Omega_{1}$ if $\Psi(0) \leqslant \Omega_{1}$ for $t \geqslant 0$, where $\Omega_{1}=\frac{\rho}{\phi}$. Since $W, U, M, N$ and $P$ are all nonnegative then $0 \leqslant W(t), U(t), M(t) \leqslant \Omega_{1}, 0 \leqslant N(t) \leqslant \Omega_{2}, 0 \leqslant P(t) \leqslant \Omega_{3}$ if $W(0)+U(0)+M(0)+\frac{a}{2 b} N(0)+\frac{\mu}{\sigma} P(0) \leqslant$ $\Omega_{1}$, where $\Omega_{2}=\frac{2 b \Omega_{1}}{a}$ and $\Omega_{3}=\frac{\sigma \Omega_{1}}{\mu}$. 


\subsection{Equilibria}

Let $(\mathrm{W}, \mathrm{U}, \mathrm{M}, \mathrm{N}, \mathrm{P})$ be any equilibrium of system (2.1) satisfying the following equations:

$$
\begin{aligned}
& 0=\rho-\alpha W-\eta_{1} W N-\eta_{2} W U-\eta_{3} W M, \\
& 0=\eta_{1} W N+\eta_{2} W U+\eta_{3} W M-(\lambda+\gamma) U, \\
& 0=\lambda U-a M-\mu P M, \\
& 0=b M-\varepsilon N, \\
& 0=(\sigma M-\pi) P .
\end{aligned}
$$

The straightforward calculation finds that system (2.1) admits three equilibria.

(1) HIV-free equilibrium, $\Xi_{0}=\left(W_{0}, 0,0,0,0\right)$, where $W_{0}=\rho / \alpha$. This case describes the situation of healthy state where the HIV infection is absent.

(2) Chronic HIV infection equilibrium with inactive CTL-mediated immune response, $\bigoplus_{1}=\left(W_{1}, U_{1}\right.$, $\left.\mathrm{M}_{1}, \mathrm{~N}_{1}, 0\right)$, where

$$
\begin{aligned}
W_{1} & =\frac{a \varepsilon(\gamma+\lambda)}{a \varepsilon \eta_{2}+\lambda\left(b \eta_{1}+\varepsilon \eta_{3}\right)}, \\
\mathrm{U}_{1} & =\frac{a \varepsilon \alpha}{a \varepsilon \eta_{2}+\lambda\left(b \eta_{1}+\varepsilon \eta_{3}\right)}\left[\frac{W_{0}\left\{a \varepsilon \eta_{2}+\lambda\left(b \eta_{1}+\varepsilon \eta_{3}\right)\right\}}{a \varepsilon(\gamma+\lambda)}-1\right], \\
M_{1} & =\frac{\varepsilon \alpha \lambda}{a \varepsilon \eta_{2}+\lambda\left(b \eta_{1}+\varepsilon \eta_{3}\right)}\left[\frac{W_{0}\left\{a \varepsilon \eta_{2}+\lambda\left(b \eta_{1}+\varepsilon \eta_{3}\right)\right\}}{a \varepsilon(\gamma+\lambda)}-1\right], \\
N_{1} & =\frac{\alpha b \lambda}{a \varepsilon \eta_{2}+\lambda\left(b \eta_{1}+\varepsilon \eta_{3}\right)}\left[\frac{W_{0}\left\{a \varepsilon \eta_{2}+\lambda\left(b \eta_{1}+\varepsilon \eta_{3}\right)\right\}}{a \varepsilon(\gamma+\lambda)}-1\right] .
\end{aligned}
$$

Therefore, $\bigoplus_{1}$ exists when

$$
\frac{W_{0}\left[a \varepsilon \eta_{2}+\lambda\left(b \eta_{1}+\varepsilon \eta_{3}\right)\right]}{a \varepsilon(\gamma+\lambda)}>1
$$

At the equilibrium $\bigoplus_{1}$ the chronic HIV infection persists while the CTL-mediated is unstimulated. The basic HIV reproduction number for system (2.1) is defined as:

$$
\mathfrak{R}_{0}=\frac{W_{0}\left[a \varepsilon \eta_{2}+\lambda\left(b \eta_{1}+\varepsilon \eta_{3}\right)\right]}{a \varepsilon(\gamma+\lambda)}=\mathfrak{R}_{01}+\mathfrak{R}_{02}+\mathfrak{R}_{03}
$$

where

$$
\mathfrak{R}_{01}=\frac{W_{0} \lambda b \eta_{1}}{a \varepsilon(\gamma+\lambda)}, \quad \mathfrak{R}_{02}=\frac{W_{0} \eta_{2}}{\gamma+\lambda}, \quad \mathfrak{R}_{03}=\frac{W_{0} \lambda \eta_{3}}{a(\gamma+\lambda)} .
$$

The parameter $\mathfrak{R}_{0}$ determines whether or not the infection will be chronic. In fact, $\mathfrak{R}_{01}$ measures the average number of secondary HIV-infected cells caused by an existing free HIV particle due to VTC transmission, while $\mathfrak{R}_{02}$ and $\mathfrak{R}_{03}$ measure the average numbers of secondary HIV-infected cells caused by living silent and active HIV-infected cell, respectively, due to CTC transmission. In terms of $\mathfrak{R}_{0}$, we can write

$$
\begin{array}{rlrl}
W_{1} & =\frac{W_{0}}{\mathfrak{R}_{0}}, & \mathrm{U}_{1} & =\frac{a \varepsilon \alpha}{a \varepsilon \eta_{2}+\lambda\left(b \eta_{1}+\varepsilon \eta_{3}\right)}\left(\Re_{0}-1\right), \\
M_{1}=\frac{\varepsilon \alpha \lambda}{a \varepsilon \eta_{2}+\lambda\left(b \eta_{1}+\varepsilon \eta_{3}\right)}\left(\Re_{0}-1\right), & N_{1}=\frac{\alpha b \lambda}{a \varepsilon \eta_{2}+\lambda\left(b \eta_{1}+\varepsilon \eta_{3}\right)}\left(\Re_{0}-1\right) .
\end{array}
$$

(3) Chronic HIV infection equilibrium with active CTL-mediated immune response,

$$
\mathrm{Ð}_{2}=\left(\mathrm{W}_{2}, \mathrm{U}_{2}, \mathrm{M}_{2}, \mathrm{~N}_{2}, \mathrm{P}_{2}\right)
$$


where

$$
W_{2}=\frac{\rho \varepsilon \sigma}{\lambda b \pi \eta_{1}+\varepsilon\left(\pi \eta_{3}+\alpha \sigma+\sigma \eta_{2} \mathrm{U}_{2}\right)}, M_{2}=\frac{\pi}{\sigma}, N_{2}=\frac{b \pi}{\varepsilon \sigma}, P_{2}=\frac{a}{\mu}\left(\frac{\lambda \sigma U_{2}}{a \pi}-1\right),
$$

and $\mathrm{U}_{2}$ satisfies the quadratic equation

$$
\tilde{\mathrm{A}} \mathrm{U}_{2}^{2}+\tilde{\mathrm{B}} \mathrm{U}_{2}+\tilde{\mathrm{C}}=0
$$

where

$$
\tilde{A}=\varepsilon \eta_{2} \sigma(\gamma+\lambda), \quad \tilde{B}=\pi\left(b \eta_{1}+\varepsilon \eta_{3}\right)(\gamma+\lambda)+\varepsilon \sigma\left[\alpha(\gamma+\lambda)-\eta_{2} \rho\right], \quad \tilde{C}=-\pi \rho\left(b \eta_{1}+\varepsilon \eta_{3}\right) .
$$

Since $\tilde{A}>0$ and $\tilde{C}<0$, then $\tilde{B}^{2}-4 \tilde{A} \tilde{C}>0$ and there are two distinct real roots of Eq. (2.4). The positive root is given by

$$
\mathrm{U}_{2}=\frac{-\tilde{\mathrm{B}}+\sqrt{\tilde{\mathrm{B}}^{2}-4 \tilde{\mathrm{A}} \tilde{C}}}{2 \tilde{\mathrm{A}}} .
$$

It follows that $W_{2}>0$ and $P_{2}>0$ only when $\frac{\lambda \sigma U_{2}}{a \pi}>1$. We define the HIV-specific CTL-mediated immunity reproduction number as follows:

$$
\mathfrak{R}_{1}=\frac{\lambda \sigma \mathrm{U}_{2}}{\mathrm{a} \pi} .
$$

Therefore, $\bigoplus_{2}$ exists when $\mathfrak{R}_{1}>1$. The parameter $\mathfrak{R}_{1}$ determines whether or not the HIV-specific CTLmediated immune response is stimulated.

\subsection{Global stability analysis}

The global stability of the equilibria will be investigated by constructing Lyapunov functional using the method presented in $[9,13,23,28]$. Let us define the function $\digamma:(0, \infty) \rightarrow[0, \infty)$ as $\digamma(v)=v-1-\ln v$. Denote $(\mathrm{W}, \mathrm{U}, \mathrm{M}, \mathrm{N}, \mathrm{P})=(\mathrm{W}(\mathrm{t}), \mathrm{U}(\mathrm{t}), \mathrm{M}(\mathrm{t}), \mathrm{N}(\mathrm{t}), \mathrm{P}(\mathrm{t}))$.

Theorem 2.2. If $\mathfrak{R}_{0} \leqslant 1$, then $\bigoplus_{0}$ is G.A.S.

Proof. Constructing a Lyapunov functional candidate:

$$
\Theta_{0}(W, U, M, N, P)=W_{0} \digamma\left(\frac{W}{W_{0}}\right)+U+\frac{W_{0}\left(b \eta_{1}+\varepsilon \eta_{3}\right)}{a \varepsilon} M+\frac{\eta_{1} W_{0}}{\varepsilon} N+\frac{\mu W_{0}\left(b \eta_{1}+\varepsilon \eta_{3}\right)}{\sigma a \varepsilon} P .
$$

It is seen that, $\Theta_{0}(W, U, M, N, P)>0$ for all $W, U, M, N, P>0$, and $\Theta_{0}$ has a global minimum at $\bigoplus_{0}$. We calculate $\frac{d \Theta_{0}}{d t}$ along the solutions of model (2.1) as:

$$
\begin{aligned}
\frac{d \Theta_{0}}{d t}= & \left(1-\frac{W_{0}}{W}\right)\left(\rho-\alpha W-\eta_{1} W N-\eta_{2} W U-\eta_{3} W M\right)+\eta_{1} W N+\eta_{2} W U+\eta_{3} W M-(\lambda+\gamma) U \\
& +\frac{W_{0}\left(b \eta_{1}+\varepsilon \eta_{3}\right)}{a \varepsilon}(\lambda U-a M-\mu P M)+\frac{\eta_{1} W_{0}}{\varepsilon}(b M-\varepsilon N)+\frac{\mu W_{0}\left(b \eta_{1}+\varepsilon \eta_{3}\right)}{\sigma a \varepsilon}(\sigma P M-\pi P) \\
= & \left(1-\frac{W_{0}}{W}\right)(\rho-\alpha W)+\eta_{2} W_{0} U-(\lambda+\gamma) U+\frac{\lambda W_{0}\left(b \eta_{1}+\varepsilon \eta_{3}\right)}{a \varepsilon} U-\frac{\mu \pi W_{0}\left(b \eta_{1}+\varepsilon \eta_{3}\right)}{\sigma a \varepsilon} P .
\end{aligned}
$$

Using $W_{0}=\rho / \alpha$, we obtain

$$
\begin{aligned}
\frac{d \Theta_{0}}{d t} & =-\alpha \frac{\left(W-W_{0}\right)^{2}}{W}+(\lambda+\gamma)\left[\frac{W_{0}\left\{a \varepsilon \eta_{2}+\lambda\left(b \eta_{1}+\varepsilon \eta_{3}\right)\right\}}{a \varepsilon(\lambda+\gamma)}-1\right] U-\frac{\mu \pi W_{0}\left(b \eta_{1}+\varepsilon \eta_{3}\right)}{\sigma a \varepsilon} P \\
& =-\alpha \frac{\left(W-W_{0}\right)^{2}}{W}+(\lambda+\gamma)\left(\Re_{0}-1\right) U-\frac{\mu \pi W_{0}\left(b \eta_{1}+\varepsilon \eta_{3}\right)}{\sigma a \varepsilon} P .
\end{aligned}
$$


Therefore, $\frac{\mathrm{d} \Theta_{0}}{\mathrm{dt}} \leqslant 0$ for all $W, \mathrm{U}, \mathrm{M}, \mathrm{N}, \mathrm{P}>0$ with equality holding when $\mathrm{W}=\mathrm{W}_{0}$ and $\mathrm{U}=\mathrm{P}=0$. The solutions of system (2.1) are confined to $\Upsilon_{0}^{\prime}$ the largest invariant subset of $\Upsilon_{0}=\left\{(W, U, M, N, P): \frac{d \Theta_{0}}{d t}=0\right\}$ [22]. The set $\Upsilon_{0}^{\prime}$ is invariant and contains elements which satisfy $W(t)=W_{0}$ and $u(t)=P(t)=0$. According to the LaSalle's invariance principle $\lim _{t \rightarrow \infty} W(t)=W_{0}$ and $\lim _{t \rightarrow \infty} U(t)=\lim _{t \rightarrow \infty} P(t)=0$. Then, $\dot{W}(t)=0$ and $\dot{\mathrm{U}}(\mathrm{t})=\dot{\mathrm{P}}(\mathrm{t})=0$. From the third and fourth equations of system (2.1), we have

$$
\dot{M}(\mathrm{t})=-\mathrm{aM}(\mathrm{t}), \quad \dot{\mathrm{N}}(\mathrm{t})=\mathrm{bM}(\mathrm{t})-\varepsilon \mathrm{N}(\mathrm{t}) .
$$

Let us define a Lyapunov function as follows:

$$
\tilde{\Theta}_{0}=M(t)+\frac{a}{2 b} N(t) .
$$

Therefore, the time derivative of $\tilde{\Theta}_{0}$ along the solutions of system (2.6) can be calculated as follows:

$$
\frac{d \tilde{\Theta}_{0}}{d t}=-\frac{a}{2}\left(M(t)+\frac{\varepsilon}{b} N(t)\right) \leqslant 0
$$

Clearly $\frac{\mathrm{d} \tilde{\Theta}_{0}}{\mathrm{~d} t}=0$ when $M(t)=N(t)=0$ for all $t$. Let $\Upsilon_{0}^{\prime \prime}=\left\{(W, U, M, N, P) \in \Upsilon_{0}^{\prime}: \frac{d \tilde{\Theta}_{0}}{d t}=0\right\}$. Thus $\Upsilon_{0}^{\prime \prime}=$ $\left\{(\mathrm{W}, \mathrm{U}, \mathrm{M}, \mathrm{N}, \mathrm{P}) \in \Upsilon_{0}^{\prime}: \mathrm{W}=\mathrm{W}_{0}, \mathrm{U}=\mathrm{M}=\mathrm{N}=\mathrm{P}=0\right\}=\left\{\bigoplus_{0}\right\}$. Hence, all solution trajectories approach $\bigoplus_{0}$ and this means that $\bigoplus_{0}$ is G.A.S [22].

Lemma 2.3. If $\Re_{1} \leqslant 1$, then $M_{1} \leqslant M_{2}$.

Proof. Let $\Re_{1} \leqslant 1$, then $\frac{\lambda \sigma \mathrm{U}_{2}}{\mathrm{a} \pi} \leqslant 1$ and hence

$$
\begin{aligned}
\mathrm{U}_{2} \leqslant \frac{a}{\lambda \sigma} \Longrightarrow \frac{-\tilde{\mathrm{B}}+\sqrt{\tilde{\mathrm{B}}^{2}-4 \tilde{\mathrm{A}} \tilde{C}}}{2 \tilde{A}} & \leqslant \frac{a \pi}{\lambda \sigma} \Longrightarrow \sqrt{\tilde{\mathrm{B}}^{2}-4 \tilde{\mathrm{A}} \tilde{\mathrm{C}}} \\
& \leqslant \frac{2 \tilde{A} a \pi+\lambda \sigma \tilde{\mathrm{B}}}{\lambda \sigma} \Longrightarrow\left(\frac{2 \tilde{\mathrm{A}} a \pi+\lambda \sigma \tilde{\mathrm{B}}}{\lambda \sigma}\right)^{2}+4 \tilde{\mathrm{A}} \tilde{\mathrm{C}}-\tilde{\mathrm{B}}^{2} \geqslant 0 .
\end{aligned}
$$

Using Eq. (2.5), we obtain

$$
\frac{4 a \pi \varepsilon \eta_{2} \sigma(\gamma+\lambda)^{2}\left[a \varepsilon \eta_{2}+\lambda\left(b \eta_{1}+\varepsilon \eta_{3}\right)\right]}{\lambda^{2}}\left(M_{2}-M_{1}\right) \geqslant 0 .
$$

Hence, $M_{1} \leqslant M_{2}$.

Theorem 2.4. If $\mathfrak{R}_{1} \leqslant 1<\mathfrak{R}_{0}$, then $\bigoplus_{1}$ is G.A.S.

Proof. Let us define a function $\Theta_{1}(\mathrm{~W}, \mathrm{U}, \mathrm{M}, \mathrm{N}, \mathrm{P})$ as:

$$
\Theta_{1}=W_{1} \digamma\left(\frac{W}{W_{1}}\right)+\mathrm{U}_{1} \digamma\left(\frac{\mathrm{U}}{\mathrm{U}_{1}}\right)+\frac{\mathrm{W}_{1}\left(\mathrm{~b} \eta_{1}+\varepsilon \eta_{3}\right)}{\mathrm{a} \varepsilon} \mathrm{M}_{1} \digamma\left(\frac{\mathrm{M}}{\mathrm{M}_{1}}\right)+\frac{\eta_{1} \mathrm{~W}_{1}}{\varepsilon} \mathrm{N}_{1} \digamma\left(\frac{\mathrm{N}}{\mathrm{N}_{1}}\right)+\frac{\mu \mathrm{W}_{1}\left(\mathrm{~b} \eta_{1}+\varepsilon \eta_{3}\right)}{\sigma a \varepsilon} \mathrm{P} .
$$

Calculating $\frac{d \Theta_{1}}{d t}$ as:

$$
\begin{aligned}
\frac{d \Theta_{1}}{d t}= & \left(1-\frac{W_{1}}{W}\right)\left(\rho-\alpha W-\eta_{1} W N-\eta_{2} W U-\eta_{3} W M\right) \\
& +\left(1-\frac{U_{1}}{\mathrm{U}}\right)\left(\eta_{1} W N+\eta_{2} W U+\eta_{3} W M-(\lambda+\gamma) \mathrm{U}\right) \\
& +\frac{W_{1}\left(b \eta_{1}+\varepsilon \eta_{3}\right)}{a \varepsilon}\left(1-\frac{M_{1}}{M}\right)(\lambda U-a M-\mu P M) \\
& +\frac{\eta_{1} W_{1}}{\varepsilon}\left(1-\frac{N_{1}}{N}\right)(b M-\varepsilon N)+\frac{\mu W_{1}\left(b \eta_{1}+\varepsilon \eta_{3}\right)}{\sigma a \varepsilon}(\sigma P M-\pi P) .
\end{aligned}
$$


Collecting terms of Eq. (2.7), we derive

$$
\begin{aligned}
\frac{d \Theta_{1}}{d t}= & \left(1-\frac{W_{1}}{W}\right)(\rho-\alpha W)+\eta_{2} W_{1} U-(\lambda+\gamma) U-\left(\eta_{1} W N+\eta_{2} W U+\eta_{3} W M\right) \frac{U_{1}}{u} \\
& +(\lambda+\gamma) U_{1}+\frac{\lambda W_{1}\left(b \eta_{1}+\varepsilon \eta_{3}\right)}{a \varepsilon} U-\frac{\lambda W_{1}\left(b \eta_{1}+\varepsilon \eta_{3}\right)}{a \varepsilon} u \frac{M_{1}}{M}+\frac{W_{1}\left(b \eta_{1}+\varepsilon \eta_{3}\right)}{\varepsilon} M_{1} \\
& +\frac{\mu W_{1}\left(b \eta_{1}+\varepsilon \eta_{3}\right)}{a \varepsilon} P_{1}-\eta_{1} W_{1} \frac{b M}{\varepsilon} \frac{N_{1}}{N}+\eta_{1} W_{1} N_{1}-\frac{\mu \pi W_{1}\left(b \eta_{1}+\varepsilon \eta_{3}\right)}{\sigma a \varepsilon} P .
\end{aligned}
$$

Using the equilibrium conditions for $\bigoplus_{1}$, we get

$$
\begin{aligned}
& \rho=\alpha W_{1}+\eta_{1} W_{1} N_{1}+\eta_{2} W_{1} U_{1}+\eta_{3} W_{1} M_{1}, \\
& \eta_{1} W_{1} N_{1}+\eta_{2} W_{1} U_{1}+\eta_{3} W_{1} M_{1}=(\lambda+\gamma) U_{1}, \\
& \frac{\lambda U_{1}}{a}=M_{1}, \quad N_{1}=\frac{b M_{1}}{\varepsilon} .
\end{aligned}
$$

In addition,

$$
\eta_{1} W_{1} N_{1}+\eta_{3} W_{1} M_{1}=\frac{W_{1}\left(b \eta_{1}+\varepsilon \eta_{3}\right)}{\varepsilon} M_{1}=\frac{\lambda W_{1}\left(b \eta_{1}+\varepsilon \eta_{3}\right)}{a \varepsilon} U_{1}
$$

Then, we obtain

$$
\begin{aligned}
\frac{d \Theta_{1}}{d t}= & \left(1-\frac{W_{1}}{W}\right)\left(\alpha W_{1}-\alpha W\right)+\left(\eta_{1} W_{1} N_{1}+\eta_{2} W_{1} U_{1}+\eta_{3} W_{1} M_{1}\right)\left(1-\frac{W_{1}}{W}\right) \\
& -\eta_{1} W_{1} N_{1} \frac{W N U_{1}}{W_{1} N_{1} U}-\eta_{2} W_{1} u_{1} \frac{W}{W_{1}}-\eta_{3} W_{1} M_{1} \frac{W M U_{1}}{W_{1} M_{1} u}+\eta_{1} W_{1} N_{1}+\eta_{2} W_{1} U_{1} \\
& +\eta_{3} W_{1} M_{1}-\left(\eta_{1} W_{1} N_{1}+\eta_{3} W_{1} M_{1}\right) \frac{u M_{1}}{U_{1} M}+\eta_{1} W_{1} N_{1}+\eta_{3} W_{1} M_{1} \\
& +\frac{\mu W_{1}\left(b \eta_{1}+\varepsilon \eta_{3}\right)}{a \varepsilon} P M_{1}-\eta_{1} W_{1} N_{1} \frac{M N_{1}}{M_{1} N}+\eta_{1} W_{1} N_{1}-\frac{\mu \pi W_{1}\left(b \eta_{1}+\varepsilon \eta_{3}\right)}{\sigma a \varepsilon} P \\
= & -\alpha \frac{(W-W)^{2}}{W}+\eta_{1} W_{1} N_{1}\left(4-\frac{W_{1}}{W}-\frac{W N U_{1}}{W_{1} N_{1} U}-\frac{u M_{1}}{U_{1} M}-\frac{M N_{1}}{M_{1} N}\right) \\
& +\eta_{2} W_{1} U_{1}\left(2-\frac{W_{1}}{W}-\frac{W}{W_{1}}\right)+\eta_{3} W_{1} M_{1}\left(3-\frac{W_{1}}{W}-\frac{W M U_{1}}{W_{1} M_{1} u}-\frac{u M_{1}}{U_{1} M}\right) \\
& +\frac{\mu W_{1}\left(b \eta_{1}+\varepsilon \eta_{3}\right)}{a \varepsilon}\left(M_{1}-\frac{\pi}{\sigma}\right) P .
\end{aligned}
$$

Therefore, Eq. (2.8) becomes

$$
\begin{aligned}
\frac{d \Theta_{1}}{d t}= & -\left(\alpha+\eta_{2} U_{1}\right) \frac{\left(W-W_{1}\right)^{2}}{W}+\eta_{1} W_{1} N_{1}\left(4-\frac{W_{1}}{W}-\frac{W N U_{1}}{W_{1} N_{1} \mathrm{U}}-\frac{u M_{1}}{U_{1} M}-\frac{M N_{1}}{M_{1} N}\right) \\
& +\eta_{3} W_{1} M_{1}\left(3-\frac{W_{1}}{W}-\frac{W M U_{1}}{W_{1} M_{1} U}-\frac{U M_{1}}{U_{1} M}\right)+\frac{\mu W_{1}\left(b \eta_{1}+\varepsilon \eta_{3}\right)}{a \varepsilon}\left(M_{1}-M_{2}\right) P .
\end{aligned}
$$

Since the arithmetical mean is greater than or equal to the geometrical mean, then

$$
\frac{W_{1}}{W}+\frac{W U_{1}}{W_{1} N_{1} U}+\frac{\mathrm{UM}_{1}}{\mathrm{U}_{1} M}+\frac{\mathrm{MN}_{1}}{\mathrm{M}_{1} \mathrm{~N}} \geqslant 4 \text { and } \frac{W_{1}}{W}+\frac{W_{M U_{1}}}{W_{1} M_{1} \mathrm{U}}+\frac{\mathrm{UM}_{1}}{\mathrm{U}_{1} M} \geqslant 3 .
$$

Thus, the second and third terms of Eq. (2.9) are less than or equal to zero. Using Lemma 2.3 and since $\mathfrak{R}_{0}>1, \mathfrak{R}_{1} \leqslant 1$ then $M_{1} \leqslant M_{2}$ and $\frac{d \Theta_{1}}{d t} \leqslant 0$ for all $W, U, M, N, P>0$ with equality holding when $W=W_{1}, U=U_{1}, M=M_{1}, N=N_{1}$ and $P=0$. Let $\Upsilon_{1}^{\prime}$ be the largest invariant subset of $\Upsilon_{1}=\left\{(W, U, M, N, P): \frac{d \Theta_{1}}{d t}=0\right\}$. It can be seen that $\Upsilon_{1}^{\prime}=\left\{\bigoplus_{1}\right\}$ and $\bigoplus_{1}$ is G.A.S using LaSalle's invariance principle. 
Theorem 2.5. For system (2.1), suppose that $\mathfrak{R}_{1}>1$, then $\bigoplus_{2}$ is G.A.S.

Proof. Define a function $\Theta_{2}(\mathrm{~W}, \mathrm{U}, \mathrm{M}, \mathrm{N}, \mathrm{P})$ as:

$$
\begin{aligned}
\Theta_{2}= & W_{2} \digamma\left(\frac{W}{W_{2}}\right)+\mathrm{U}_{2} \digamma\left(\frac{\mathrm{U}}{\mathrm{U}_{2}}\right)+\frac{\mathrm{W}_{2}\left(b \eta_{1}+\varepsilon \eta_{3}\right)}{\varepsilon\left(\mathrm{a}+\mu \mathrm{P}_{2}\right)} M_{2} \digamma\left(\frac{M}{M_{2}}\right) \\
& +\frac{\eta_{1} W_{2}}{\varepsilon} \mathrm{N}_{2} \digamma\left(\frac{N}{N_{2}}\right)+\frac{\mu W_{2}\left(b \eta_{1}+\varepsilon \eta_{3}\right)}{\sigma \varepsilon\left(a+\mu P_{2}\right)} P_{2} \digamma\left(\frac{P}{P_{2}}\right) .
\end{aligned}
$$

We calculate $\frac{\mathrm{d} \Theta_{2}}{\mathrm{dt}}$ as:

$$
\begin{aligned}
\frac{d \Theta_{2}}{d t}= & \left(1-\frac{W_{2}}{W}\right)\left(\rho-\alpha W-\eta_{1} W N-\eta_{2} W U-\eta_{3} W M\right) \\
& +\left(1-\frac{U_{2}}{U}\right)\left(\eta_{1} W N+\eta_{2} W U+\eta_{3} W M-(\lambda+\gamma) U\right) \\
& +\frac{W_{2}\left(b \eta_{1}+\varepsilon \eta_{3}\right)}{\varepsilon\left(a+\mu P_{2}\right)}\left(1-\frac{M_{2}}{M}\right)(\lambda U-a M-\mu P M) \\
& +\frac{\eta_{1} W_{2}}{\varepsilon}\left(1-\frac{N_{2}}{N}\right)(b M-\varepsilon N)+\frac{\mu W_{2}\left(b \eta_{1}+\varepsilon \eta_{3}\right)}{\sigma \varepsilon\left(a+\mu P_{2}\right)}\left(1-\frac{P_{2}}{P}\right)(\sigma P M-\pi P) .
\end{aligned}
$$

Collecting terms of Eq. (2.10), we derive

$$
\begin{aligned}
\frac{d \Theta_{2}}{d t}= & \left(1-\frac{W_{2}}{W}\right)(\rho-\alpha W)+\eta_{2} W_{2} U+\eta_{3} W_{2} M-(\lambda+\gamma) U-\eta_{1} W N \frac{U_{2}}{u}-\eta_{2} W U_{2}-\eta_{3} W M \frac{U_{2}}{u} \\
& +(\lambda+\gamma) U_{2}+\frac{\lambda W_{2}\left(b \eta_{1}+\varepsilon \eta_{3}\right)}{\varepsilon\left(a+\mu P_{2}\right)} U-\frac{a W_{2}\left(b \eta_{1}+\varepsilon \eta_{3}\right)}{\varepsilon\left(a+\mu P_{2}\right)} M-\frac{\lambda W_{2}\left(b \eta_{1}+\varepsilon \eta_{3}\right)}{\varepsilon\left(a+\mu P_{2}\right)} U \frac{M_{2}}{M} \\
& +\frac{a W_{2}\left(b \eta_{1}+\varepsilon \eta_{3}\right)}{\varepsilon\left(a+\mu P_{2}\right)} M_{2}+\frac{\mu W_{2}\left(b \eta_{1}+\varepsilon \eta_{3}\right)}{\varepsilon\left(a+\mu P_{2}\right)} P M_{2}+\frac{\eta_{1} W_{2}}{\varepsilon} b M-\frac{\eta_{1} W_{2}}{\varepsilon} b M \frac{N_{2}}{N}+\eta_{1} W_{2} N_{2} \\
& -\frac{\mu \pi W_{2}\left(b \eta_{1}+\varepsilon \eta_{3}\right)}{\sigma \varepsilon\left(a+\mu P_{2}\right)} P-\frac{\mu W_{2}\left(b \eta_{1}+\varepsilon \eta_{3}\right)}{\varepsilon\left(a+\mu P_{2}\right)} P_{2} M+\frac{\mu \pi W_{2}\left(b \eta_{1}+\varepsilon \eta_{3}\right)}{\sigma \varepsilon\left(a+\mu P_{2}\right)} P_{2} .
\end{aligned}
$$

Using the equilibrium conditions for $\bigoplus_{2}$ :

$$
\begin{aligned}
& \rho=\alpha W_{2}+\eta_{1} W_{2} N_{2}+\eta_{2} W_{2} U_{2}+\eta_{3} W_{2} M_{2} \\
& \eta_{1} W_{2} N_{2}+\eta_{2} W_{2} U_{2}+\eta_{3} W_{2} M_{2}=(\lambda+\gamma) U_{2} \\
& \lambda U_{2}=\left(a+\mu P_{2}\right) M_{2}, \quad M_{2}=\frac{\pi}{\sigma}, \quad N_{2}=\frac{b}{\varepsilon} M_{2} .
\end{aligned}
$$

Further,

$$
\eta_{1} W_{2} N_{2}+\eta_{3} W_{2} M_{2}=\frac{W_{2}\left(b \eta_{1}+\varepsilon \eta_{3}\right)}{\varepsilon} M_{2}=\frac{\lambda W_{2}\left(b \eta_{1}+\varepsilon \eta_{3}\right)}{\varepsilon\left(a+\mu P_{2}\right)} U_{2}
$$

Therefore, we obtain

$$
\begin{aligned}
\frac{d \Theta_{2}}{d t}= & \left(1-\frac{W_{2}}{W}\right)\left(\alpha W_{2}-\alpha W\right)+\left(\eta_{1} W_{2} N_{2}+\eta_{2} W_{2} U_{2}+\eta_{3} W_{2} M_{2}\right)\left(1-\frac{W_{2}}{W}\right) \\
& -\eta_{1} W_{2} N_{2} \frac{W U_{2}}{W_{2} N_{2} U}-\eta_{2} W_{2} U_{2} \frac{W}{W_{2}}-\eta_{2} W_{2} M_{2} \frac{W M U_{2}}{W_{2} M_{2} U}+\eta_{1} W_{2} N_{2}+\eta_{2} W_{2} U_{2}+\eta_{3} W_{2} M_{2} \\
& -\left(\eta_{1} W_{2} N_{2}+\eta_{3} W_{2} M_{2}\right) \frac{U_{2}}{U_{2} M}+\eta_{1} W_{2} N_{2}+\eta_{3} W_{2} M_{2}-\eta_{1} W_{2} N_{2} \frac{M N_{2}}{M_{2} N}+\eta_{1} W_{2} N_{2} \\
= & -\alpha \frac{\left(W-W_{2}\right)^{2}}{W}+\eta_{1} W_{2} N_{2}\left(4-\frac{W_{2}}{W}-\frac{W N U_{2}}{W_{2} N_{2} U}-\frac{U M_{2}}{U_{2} M}-\frac{M N_{2}}{M_{2} N}\right)
\end{aligned}
$$




$$
\begin{aligned}
& +\eta_{2} W_{2} u_{2}\left(2-\frac{W_{2}}{W}-\frac{W}{W_{2}}\right)+\eta_{3} W_{2} M_{2}\left(3-\frac{W_{2}}{W}-\frac{W u_{2}}{W_{2} M_{2} u}-\frac{u_{2}}{u_{2} M}\right) \\
& =-\left(\alpha+\eta_{2} \mathrm{U}_{2}\right) \frac{\left(\mathrm{W}-\mathrm{W}_{2}\right)^{2}}{W}+\eta_{1} \mathrm{~W}_{2} \mathrm{~N}_{2}\left(4-\frac{W_{2}}{W}-\frac{W \mathrm{NU}_{2}}{W_{2} \mathrm{~N}_{2} \mathrm{U}}-\frac{\mathrm{UM}_{2}}{\mathrm{U}_{2} M}-\frac{\mathrm{MN}_{2}}{M_{2} \mathrm{~N}}\right) \\
& +\eta_{3} W_{2} M_{2}\left(3-\frac{W_{2}}{W}-\frac{W_{M U}}{W_{2} M_{2} U}-\frac{u_{2}}{U_{2} M}\right) \text {. }
\end{aligned}
$$

Hence, if $\Re_{1}>1$, then $\frac{d \Theta_{2}}{d t} \leqslant 0$ for all $W, U, M, N, P>0$. Similar to the previous Theorems one can show that $\frac{\mathrm{d} \Theta_{2}}{\mathrm{dt}}=0$ when $W=W_{2}, U=U_{2}, M=M_{2}$ and $N=N_{2}$. The solutions of system (2.1) tend to $\Upsilon_{2}^{\prime}$ the largest invariant subset of $\Upsilon_{2}=\left\{(W, U, M, N, P): \frac{d \Theta_{2}}{d t}=0\right\}$. For each element of $\Upsilon_{2}^{\prime}$ we have $\mathrm{W}(\mathrm{t})=\mathrm{W}_{2}, \mathrm{U}(\mathrm{t})=\mathrm{U}_{2}, \mathrm{M}(\mathrm{t})=\mathrm{M}_{2}, \mathrm{~N}(\mathrm{t})=\mathrm{N}_{2}$, then $\dot{M}(\mathrm{t})=0$ and from the third equation of system (2.1) we have $0=\dot{M}(t)=\lambda U_{2}-a_{M}-\mu P(t) M_{2}$, which gives $\mathrm{P}(\mathrm{t})=\mathrm{P}_{2}$ for all $t$. Therefore, $\Upsilon_{2}^{\prime}=\left\{\mathrm{\Xi}_{2}\right\}$. Applying LaSalle's invariance principle we get $\mathrm{Ð}_{2}$ is G.A.S.

\section{Model with saturated infection rates}

It is well known that model with saturated infection rates is much more accurate to describe the VTC and CTC transmissions in case of the high concentration of virus particles, silent HIV-infected cells or active HIV-infected cells. Thus, in this section we focus on the following system of ordinary differential equations:

$$
\left\{\begin{array}{l}
\dot{W}(t)=\rho-\alpha W(t)-\frac{\eta_{1} W(t) N(t)}{1+\beta_{1} N(t)}-\frac{\eta_{2} W(t) U(t)}{1+\beta_{2} U(t)}-\frac{\eta_{3} W(t) M(t)}{1+\beta_{3} M(t)} \\
\dot{U}(t)=\frac{\eta_{1} W(t) N(t)}{1+\beta_{1} N(t)}+\frac{\eta_{2} W(t) U(t)}{1+\beta_{2} U(t)}+\frac{\eta_{3} W(t) M(t)}{1+\beta_{3} M(t)}-\lambda U(t)-\gamma U(t) \\
\dot{M}(t)=\lambda U(t)-a M(t)-\mu P(t) M(t) \\
\dot{N}(t)=b M(t)-\varepsilon N(t) \\
\dot{P}(t)=\sigma P(t) M(t)-\pi P(t)
\end{array}\right.
$$

where $\beta_{i}, i=1,2,3$ are the saturation constants. All other variables and parameters have the same biological interpretations that described in the previous sections. Similar to the proof of Proposition 2.1 one can easily show that $Q$ defined in (2.2) is positively invariant for system (3.1).

\subsection{Equilibria}

Let $(\bar{W}, \overline{\mathrm{U}}, \overline{\mathrm{M}}, \overline{\mathrm{N}}, \overline{\mathrm{P}})$ be any equilibrium of system (3.1) satisfying the following equations:

$$
\begin{aligned}
& 0=\rho-\alpha \bar{W}-\frac{\eta_{1} \bar{W} \bar{N}}{1+\beta_{1} \bar{N}}-\frac{\eta_{2} \bar{W} \bar{U}}{1+\beta_{2} \bar{U}}-\frac{\eta_{3} \bar{W} \bar{M}}{1+\beta_{3} \bar{M}^{\prime}} \\
& 0=\frac{\eta_{1} \bar{W} \bar{N}}{1+\beta_{1} \bar{N}}+\frac{\eta_{2} \bar{W} \bar{U}}{1+\beta_{2} \bar{U}}+\frac{\eta_{3} \bar{W} \bar{M}}{1+\beta_{3} \bar{M}}-(\lambda+\gamma) \bar{U} \\
& 0=\lambda \bar{U}-a \bar{M}-\mu \bar{P} \bar{M}, \\
& 0=b \bar{M}-\varepsilon \bar{N} \\
& 0=(\sigma \bar{M}-\pi) \bar{P} .
\end{aligned}
$$

From Eq. (3.6) we have two possibilities $\overline{\mathrm{P}}=0$ and $\bar{M}=\frac{\pi}{\sigma}$ :

(i) When $\bar{P}=0$, then from Eqs. (3.2), (3.4), and (3.5) we get

$$
\bar{W}=\rho /\left[\alpha+\left(\frac{\eta_{2}}{1+\beta_{2} \bar{u}}+\frac{b \lambda \eta_{1}}{a \varepsilon+b \lambda \beta_{1} \bar{u}}+\frac{\lambda \eta_{3}}{a+\lambda \beta_{3} \bar{u}}\right) \bar{u}\right], \quad \bar{M}=\frac{\lambda \bar{u}}{a}, \quad \bar{N}=\frac{b \lambda \bar{u}}{a \varepsilon} .
$$


Substituting the above values in Eq. (3.3), we get

$$
\overline{\mathrm{U}}\left(\check{\mathrm{A}} \overline{\mathrm{U}}^{3}+\check{\mathrm{B}} \overline{\mathrm{U}}^{2}+\check{\mathrm{C}} \overline{\mathrm{U}}+\check{\mathrm{D}}\right)=0,
$$

where

$$
\begin{aligned}
\check{\mathrm{A}}= & b \lambda^{2}(\gamma+\lambda)\left(\alpha \beta_{1} \beta_{2} \beta_{3}+\beta_{2} \beta_{3} \eta_{1}+\beta_{1} \beta_{3} \eta_{2}+\beta_{1} \beta_{2} \eta_{3}\right), \\
\check{\mathrm{B}}= & b \lambda \beta_{1} \beta_{2}\left[a \alpha(\gamma+\lambda)-\eta_{3} \lambda \rho\right]+\lambda \beta_{2} \beta_{3}\left[a \alpha \varepsilon(\gamma+\lambda)-b \eta_{1} \lambda \rho\right]+b \lambda^{2} \beta_{1} \beta_{3}\left(\alpha \gamma-\eta_{2} \rho\right) \\
& +\lambda\left[(\gamma+\lambda)\left\{a b\left(\beta_{2} \eta_{1}+\beta_{1} \eta_{2}\right)+a \varepsilon\left(\beta_{3} \eta_{2}+\beta_{2} \eta_{3}\right)+b \lambda\left(\beta_{3} \eta_{1}+\beta_{1} \eta_{3}\right)\right\}+b \alpha \beta_{1} \beta_{3} \lambda^{2}\right], \\
\check{C}= & a \beta_{2}\left[\varepsilon a \alpha(\gamma+\lambda)-\lambda \rho\left(b \eta_{1}+\varepsilon \eta_{3}\right)\right]+b \beta_{1} \lambda\left[a \alpha(\gamma+\lambda)-\rho\left(a \eta_{2}+\eta_{3} \lambda\right)\right] \\
& +\beta_{3} \lambda\left[a \alpha \varepsilon(\gamma+\lambda)-\rho\left(a \varepsilon \eta_{2}+b \eta_{1} \lambda\right)\right]+a(\gamma+\lambda)\left[a \varepsilon \eta_{2}+\lambda\left(b \eta_{1}+\varepsilon \eta_{3}\right)\right], \\
\check{D}= & -a^{2} \alpha \varepsilon(\gamma+\lambda)\left[\frac{\bar{W}_{0}\left\{a \varepsilon \eta_{2}+\lambda\left(b \eta_{1}+\varepsilon \eta_{3}\right)\right\}}{a \varepsilon(\gamma+\lambda)}-1\right] .
\end{aligned}
$$

Eq. (3.7) has two solutions $\bar{U}=0$ which gives the HIV-free equilibrium, $\bar{Ð}_{0}=\left(\bar{W}_{0}, 0,0,0,0\right)$, where $\bar{W}_{0}=\rho / \alpha$. This case describes the situation of healthy state where the HIV infection is absent. The other solution of Eq. (3.7) is

$$
\check{A} \bar{U}^{3}+\check{B} \bar{U}^{2}+\check{C} \bar{U}+\check{D}=0 .
$$

Let us define

$$
\mathcal{P}(\overline{\mathrm{U}})=\check{\mathrm{A}} \overline{\mathrm{U}}^{3}+\check{\mathrm{B}} \overline{\mathrm{U}}^{2}+\check{\mathrm{C}} \overline{\mathrm{U}}+\check{\mathrm{D}}=0 .
$$

Clearly, $\mathcal{P}(0)=\check{\mathrm{D}}<0$ if and only if $\frac{\bar{W}_{0}\left[a \varepsilon \eta_{2}+\lambda\left(b \eta_{1}+\varepsilon \eta_{3}\right)\right]}{a \varepsilon(\gamma+\lambda)}>1$. Moreover, we have $\lim _{\overline{\mathrm{U}} \rightarrow \infty} \mathcal{P}(\overline{\mathrm{U}})=\infty$. The intermediate value property of continuous functions implies that there exists $\overline{\mathrm{U}}_{1} \in(0, \infty)$ such that $\mathcal{P}\left(\overline{\mathrm{U}}_{1}\right)=0$. Therefore,

$$
\bar{W}_{1}=\rho /\left[\alpha+\left(\frac{\eta_{2}}{1+\beta_{2} \bar{U}_{1}}+\frac{b \lambda \eta_{1}}{a \varepsilon+b \lambda \beta_{1} \bar{U}_{1}}+\frac{\lambda \eta_{3}}{a+\lambda \beta_{3} \bar{U}_{1}}\right) \bar{U}_{1}\right]>0, \quad \bar{M}_{1}=\frac{\lambda \bar{U}_{1}}{a}>0, \quad \bar{N}_{1}=\frac{b \lambda \bar{U}_{1}}{a \varepsilon}>0 .
$$

Hence, a chronic HIV infection equilibrium with inactive CTL-mediated immune response, $\bar{Ð}_{1}=\left(\overline{\mathrm{W}}_{1}, \overline{\mathrm{U}}_{1}\right.$, $\left.\bar{M}_{1}, \bar{N}_{1}, 0\right)$ exists when

$$
\frac{\bar{W}_{0}\left[a \varepsilon \eta_{2}+\lambda\left(b \eta_{1}+\varepsilon \eta_{3}\right)\right]}{a \varepsilon(\gamma+\lambda)}>1
$$

At the equilibrium $\bar{Ð}_{1}$ the chronic HIV infection persists while the CTL-mediated immune response is unstimulated. The basic HIV reproduction number for system (3.1) is defined as:

$$
\overline{\mathfrak{R}}_{0}=\frac{\bar{W}_{0}\left[\mathrm{a} \varepsilon \eta_{2}+\lambda\left(b \eta_{1}+\varepsilon \eta_{3}\right)\right]}{a \varepsilon(\gamma+\lambda)}=\overline{\mathfrak{R}}_{01}+\overline{\mathfrak{R}}_{02}+\overline{\mathfrak{R}}_{03}
$$

where

$$
\overline{\mathfrak{R}}_{01}=\frac{\bar{W}_{0} \lambda \mathrm{b} \eta_{1}}{\mathrm{a} \varepsilon(\gamma+\lambda)}, \quad \overline{\mathfrak{R}}_{02}=\frac{\bar{W}_{0} \eta_{2}}{\gamma+\lambda}, \quad \overline{\mathfrak{R}}_{03}=\frac{\bar{W}_{0} \lambda \eta_{3}}{\mathrm{a}(\gamma+\lambda)} .
$$

Note that since $\bar{W}_{0}=W_{0}$, then $\overline{\mathfrak{R}}_{0}=\mathfrak{R}_{0}$, where $\mathfrak{R}_{0}$ is given by Eq. (2.3). Therefore, the saturation does not change the parameter $\mathfrak{R}_{0}$.

(ii) When $\bar{P} \neq 0$, the system has a chronic HIV infection equilibrium with active CTL-mediated immune response, $\bar{Ð}_{2}=\left(\overline{\mathrm{W}}_{2}, \overline{\mathrm{U}}_{2}, \overline{\mathrm{M}}_{2}, \overline{\mathrm{N}}_{2}, \overline{\mathrm{P}}_{2}\right)$, where

$$
\bar{W}_{2}=\rho /\left(\alpha+\frac{\eta_{2} \overline{\mathrm{U}}_{2}}{1+\beta_{2} \overline{\mathrm{U}}_{2}}+\frac{\pi \eta_{3}}{\pi \beta_{3}+\sigma}+\frac{\mathrm{b} \pi \eta_{1}}{\mathrm{~b} \pi \beta_{1}+\varepsilon \sigma}\right), \quad \bar{M}_{2}=\frac{\pi}{\sigma}, \overline{\mathrm{N}}_{2}=\frac{\mathrm{b} \pi}{\varepsilon \sigma}, \overline{\mathrm{P}}_{2}=\frac{\mathrm{a}}{\mu}\left(\frac{\lambda \sigma \overline{\mathrm{U}}_{2}}{\mathrm{a} \pi}-1\right),
$$


and $\overline{\mathrm{U}}_{2}$ satisfies the quadratic equation

$$
\hat{A} \bar{U}_{2}^{2}+\hat{B} \bar{U}_{2}+\hat{C}=0
$$

where

$$
\begin{aligned}
\hat{\mathrm{A}}= & (\gamma+\lambda)\left[\mathrm{b} \pi^{2}\left(\alpha \beta_{1} \beta_{2} \beta_{3}+\beta_{2} \beta_{3} \eta_{1}+\beta_{1} \beta_{3} \eta_{2}+\beta_{1} \beta_{2} \eta_{3}\right)\right. \\
& \left.+\pi \varepsilon \sigma\left(\alpha \beta_{2} \beta_{3}+\beta_{2} \eta_{3}+\beta_{3} \eta_{2}\right)+b \pi \sigma\left(\beta_{2} \eta_{1}+\beta_{1} \eta_{2}+\alpha \beta_{1} \beta_{2}\right)+\varepsilon \sigma^{2}\left(\alpha \beta_{2}+\eta_{2}\right)\right], \\
\hat{\mathrm{B}}= & \pi \beta_{3}\left[\alpha(\gamma+\lambda)-\eta_{2} \rho\right]\left(b \pi \beta_{1}+\varepsilon \sigma\right)+b \pi \beta_{1}\left[(\gamma+\lambda)\left(\pi \eta_{3}+\alpha \sigma\right)-\eta_{2} \rho \sigma\right] \\
& +b \pi^{2}\left[\beta_{3} \eta_{1}(\gamma+\lambda)-\beta_{2} \rho\left(\beta_{3} \eta_{1}+\beta_{1} \eta_{3}\right)\right]-\pi \beta_{2} \rho \sigma\left(b \eta_{1}+\varepsilon \eta_{3}\right) \\
& +\sigma(\gamma+\lambda)\left[\pi\left(b \eta_{1}+\varepsilon \eta_{3}\right)+\alpha \varepsilon \sigma\right]-\varepsilon \eta_{2} \rho \sigma^{2}, \\
\hat{C}= & -\pi \rho\left[b \pi\left(\beta_{3} \eta_{1}+\beta_{1} \eta_{3}\right)+\sigma\left(b \eta_{1}+\varepsilon \eta_{3}\right)\right] .
\end{aligned}
$$

Since $\hat{A}>0$ and $\hat{C}<0$, then $\hat{B}^{2}-4 \hat{A} \hat{C}>0$ and there are two distinct real roots of Eq. (3.8). The positive root is given by

$$
\overline{\mathrm{U}}_{2}=\frac{-\hat{\mathrm{B}}+\sqrt{\hat{\mathrm{B}}^{2}-4 \hat{\mathrm{A}} \hat{C}}}{2 \hat{\mathrm{A}}} .
$$

It follows that $\bar{W}_{2}>0$ and $\bar{P}_{2}>0$ only when $\frac{\lambda \sigma \bar{U}_{2}}{a \pi}>1$. We define the HIV specific CTL-mediated immunity reproduction number as follows:

$$
\overline{\mathfrak{R}}_{1}=\frac{\lambda \sigma \overline{\mathrm{U}}_{2}}{\mathrm{a} \pi} .
$$

Therefore, $\bar{Ð}_{2}$ exists when $\overline{\mathfrak{R}}_{1}>1$. The parameter $\overline{\mathfrak{R}}_{1}$ determines whether or not the HIV-specific CTLmediated immune response is stimulated.

\subsection{Global stability analysis}

The global stability of the equilibria will be investigated by constructing Lyapunov functional using the method presented in Subsection 2.3.

Theorem 3.1. If $\overline{\mathfrak{R}}_{0} \leqslant 1$, then $\bar{Ð}_{0}$ is G.A.S.

Proof. Constructing a Lyapunov function candidate:

$$
\bar{\Theta}_{0}(W, U, M, N, P)=\bar{W}_{0} \digamma\left(\frac{W}{\bar{W}_{0}}\right)+U+\frac{\bar{W}_{0}\left(b \eta_{1}+\varepsilon \eta_{3}\right)}{a \varepsilon} M+\frac{\eta_{1} \bar{W}_{0}}{\varepsilon} N+\frac{\mu \bar{W}_{0}\left(b \eta_{1}+\varepsilon \eta_{3}\right)}{\sigma a \varepsilon} P .
$$

We calculate $\frac{\mathrm{d} \bar{\Theta}_{0}}{\mathrm{dt}}$ as:

$$
\begin{aligned}
\frac{\mathrm{d} \bar{\Theta}_{0}}{\mathrm{dt}}= & \left(1-\frac{\bar{W}_{0}}{W}\right)\left(\rho-\alpha W-\frac{\eta_{1} W N}{1+\beta_{1} N}-\frac{\eta_{2} W U}{1+\beta_{2} \mathrm{U}}-\frac{\eta_{3} W M}{1+\beta_{3} M}\right)+\frac{\eta_{1} W N}{1+\beta_{1} N}+\frac{\eta_{2} W U}{1+\beta_{2} \mathrm{U}} \\
& +\frac{\eta_{3} W M}{1+\beta_{3} M}-(\lambda+\gamma) \mathrm{U}+\frac{\bar{W}_{0}\left(b \eta_{1}+\varepsilon \eta_{3}\right)}{a \varepsilon}(\lambda U-a M-\mu P M)+\frac{\eta_{1} \bar{W}_{0}}{\varepsilon}(b M-\varepsilon N) \\
& +\frac{\mu \bar{W}_{0}\left(b \eta_{1}+\varepsilon \eta_{3}\right)}{\sigma a \varepsilon}(\sigma P M-\pi P) \\
= & \left(1-\frac{\bar{W}_{0}}{W}\right)(\rho-\alpha W)+\frac{\eta_{1} \bar{W}_{0} N}{1+\beta_{1} N}+\frac{\eta_{2} \bar{W}_{0} \mathrm{U}}{1+\beta_{2} \mathrm{U}}+\frac{\eta_{3} \bar{W}_{0} M}{1+\beta_{3} M}-(\lambda+\gamma) \mathrm{U} \\
& +\frac{\lambda \bar{W}_{0}\left(b \eta_{1}+\varepsilon \eta_{3}\right)}{a \varepsilon} \mathrm{U}-\frac{\bar{W}_{0}\left(b \eta_{1}+\varepsilon \eta_{3}\right)}{\varepsilon} M+\frac{\eta_{1} \bar{W}_{0}}{\varepsilon} b M-\eta_{1} \bar{W}_{0} N-\frac{\mu \pi \bar{W}_{0}\left(b \eta_{1}+\varepsilon \eta_{3}\right)}{\sigma a \varepsilon} P .
\end{aligned}
$$


After some calculation and using $\bar{W}_{0}=\rho / \alpha$, we get

$$
\begin{aligned}
\frac{\mathrm{d} \bar{\Theta}_{0}}{\mathrm{dt}}= & -\alpha \frac{\left(W-\bar{W}_{0}\right)^{2}}{W}-\frac{\beta_{1} \eta_{1} \bar{W}_{0}}{1+\beta_{1} N} N^{2}-\frac{\beta_{2} \eta_{2} \bar{W}_{0}}{1+\beta_{2} \mathrm{u}} \mathrm{U}^{2}-\frac{\beta_{3} \eta_{3} \bar{W}_{0}}{1+\beta_{3} M} M^{2} \\
& +(\lambda+\gamma)\left[\frac{\bar{W}_{0}\left\{a \varepsilon \eta_{2}+\lambda\left(b \eta_{1}+\varepsilon \eta_{3}\right)\right\}}{a \varepsilon(\lambda+\gamma)}-1\right] \mathrm{U}-\frac{\mu \pi \bar{W}_{0}\left(b \eta_{1}+\varepsilon \eta_{3}\right)}{\sigma a \varepsilon} \mathrm{P} \\
= & -\alpha \frac{\left(W-\bar{W}_{0}\right)^{2}}{W}-\frac{\beta_{1} \eta_{1} \bar{W}_{0}}{1+\beta_{1} N} N^{2}-\frac{\beta_{2} \eta_{2} \bar{W}_{0}}{1+\beta_{2} \mathrm{U}} \mathrm{U}^{2}-\frac{\beta_{3} \eta_{3} \bar{W}_{0}}{1+\beta_{3} M} M^{2} \\
& +(\lambda+\gamma)\left(\overline{\mathfrak{R}}_{0}-1\right) \mathrm{U}-\frac{\mu \pi \bar{W}_{0}\left(b \eta_{1}+\varepsilon \eta_{3}\right)}{\sigma a \varepsilon} \mathrm{P} .
\end{aligned}
$$

Therefore, $\frac{d \bar{\Theta}_{0}}{d t} \leqslant 0$ for all $W, U, M, N, P>0$. Let $\bar{\Upsilon}_{0}=\left\{(W, U, M, N, P): \frac{d \bar{\Theta}_{0}}{d t}=0\right\}$ and $\bar{\Upsilon}_{0}^{\prime}$ be the largest invariant subset of $\bar{\Upsilon}_{0}$. The solutions of system (3.1) are converged to $\bar{\Upsilon}_{0}^{\prime}$. It is clear from Eq. (3.9) that $\frac{\mathrm{d} \bar{\Theta}_{0}}{\mathrm{dt}}=0$ when $\mathrm{W}=\bar{W}_{0}$ and $\mathrm{U}=\mathrm{M}=\mathrm{N}=\mathrm{P}=0$. Thus, LaSalle's invariance principle implies that $\bar{\Xi}_{0}$ is G.A.S.

Theorem 3.2. Suppose that $\overline{\mathfrak{R}}_{1} \leqslant 1<\overline{\mathfrak{R}}_{0}$, then $\bar{\emptyset}_{1}$ is G.A.S.

Proof. Let us define a function $\bar{\Theta}_{1}(\mathrm{~W}, \mathrm{U}, \mathrm{M}, \mathrm{N}, \mathrm{P})$ as:

$$
\begin{aligned}
\bar{\Theta}_{1}= & \bar{W}_{1} \digamma\left(\frac{W}{\bar{W}_{1}}\right)+\overline{\mathrm{U}}_{1} \digamma\left(\frac{\mathrm{U}}{\overline{\mathrm{U}}_{1}}\right)+\frac{\bar{W}_{1}\left[\mathrm{~b} \eta_{1}\left(1+\beta_{3} \overline{\mathrm{M}}_{1}\right)+\varepsilon \eta_{3}\left(1+\beta_{1} \overline{\mathrm{N}}_{1}\right)\right]}{\mathrm{a} \varepsilon\left(1+\beta_{1} \overline{\mathrm{N}}_{1}\right)\left(1+\beta_{3} \overline{\mathrm{M}}_{1}\right)} \overline{\mathrm{M}}_{1} \digamma\left(\frac{\mathrm{M}}{\overline{\mathrm{M}}_{1}}\right) \\
& +\frac{\eta_{1} \bar{W}_{1}}{\varepsilon\left(1+\beta_{1} \overline{\mathrm{N}}_{1}\right)} \overline{\mathrm{N}}_{1} \digamma\left(\frac{\mathrm{N}}{\overline{\mathrm{N}}_{1}}\right)+\frac{\mu \bar{W}_{1}\left[\mathrm{~b} \eta_{1}\left(1+\beta_{3} \bar{M}_{1}\right)+\varepsilon \eta_{3}\left(1+\beta_{1} \overline{\mathrm{N}}_{1}\right)\right]}{\sigma a \varepsilon\left(1+\beta_{1} \overline{\mathrm{N}}_{1}\right)\left(1+\beta_{3} \overline{\mathrm{M}}_{1}\right)} \mathrm{P} .
\end{aligned}
$$

Calculating $\frac{\mathrm{d} \bar{\Theta}_{1}}{\mathrm{dt}}$ as:

$$
\begin{aligned}
\frac{\mathrm{d} \bar{\Theta}_{1}}{\mathrm{dt}}= & \left(1-\frac{\bar{W}_{1}}{\mathrm{~W}}\right)\left(\rho-\alpha \mathrm{W}-\frac{\eta_{1} W \mathrm{~N}}{1+\beta_{1} \mathrm{~N}}-\frac{\eta_{2} W \mathrm{U}}{1+\beta_{2} \mathrm{U}}-\frac{\eta_{3} W M}{1+\beta_{3} M}\right) \\
& +\left(1-\frac{\overline{\mathrm{U}}_{1}}{\mathrm{U}}\right)\left(\frac{\eta_{1} W \mathrm{~N}}{1+\beta_{1} \mathrm{~N}}+\frac{\eta_{2} W \mathrm{U}}{1+\beta_{2} \mathrm{U}}+\frac{\eta_{3} W M}{1+\beta_{3} M}-(\lambda+\gamma) \mathrm{U}\right) \\
& +\frac{\bar{W}_{1}\left[\mathrm{~b} \eta_{1}\left(1+\beta_{3} \bar{M}_{1}\right)+\varepsilon \eta_{3}\left(1+\beta_{1} \overline{\mathrm{N}}_{1}\right)\right]}{a \varepsilon\left(1+\beta_{1} \overline{\mathrm{N}}_{1}\right)\left(1+\beta_{3} \bar{M}_{1}\right)}\left(1-\frac{\bar{M}_{1}}{M}\right)(\lambda \mathrm{U}-\mathrm{a} M-\mu \mathrm{PM}) \\
& +\frac{\eta_{1} \bar{W}_{1}}{\varepsilon\left(1+\beta_{1} \overline{\mathrm{N}}_{1}\right)}\left(1-\frac{\overline{\mathrm{N}}_{1}}{\mathrm{~N}}\right)(\mathrm{bM}-\varepsilon \mathrm{N}) \\
& +\frac{\mu \bar{W}_{1}\left[\mathrm{~b} \eta_{1}\left(1+\beta_{3} \bar{M}_{1}\right)+\varepsilon \eta_{3}\left(1+\beta_{1} \overline{\mathrm{N}}_{1}\right)\right]}{\sigma a \varepsilon\left(1+\beta_{1} \overline{\mathrm{N}}_{1}\right)\left(1+\beta_{3} \bar{M}_{1}\right)}(\sigma \mathrm{PM}-\pi \mathrm{P})
\end{aligned}
$$

Collecting terms of Eq. (3.10), we derive

$$
\begin{aligned}
& \frac{d \bar{\Theta}_{1}}{d t}=\left(1-\frac{\bar{W}_{1}}{W}\right)(\rho-\alpha W)+\frac{\eta_{1} \bar{W}_{1} N}{1+\beta_{1} N}+\frac{\eta_{2} \bar{W}_{1} \mathrm{U}}{1+\beta_{2} \mathrm{U}}+\frac{\eta_{3} \bar{W}_{1} M}{1+\beta_{3} M}-(\lambda+\gamma) \mathrm{U}-\frac{\eta_{1} W N}{1+\beta_{1} N} \frac{\bar{U}_{1}}{\mathrm{U}} \\
& -\frac{\eta_{2} W U}{1+\beta_{2} \mathrm{U}} \frac{\overline{\mathrm{U}}_{1}}{\mathrm{U}}-\frac{\eta_{3} W M}{1+\beta_{3} M} \frac{\overline{\mathrm{U}}_{1}}{\mathrm{U}}+(\lambda+\gamma) \overline{\mathrm{U}}_{1}+\frac{\lambda \bar{W}_{1}\left[\mathrm{~b} \eta_{1}\left(1+\beta_{3} \bar{M}_{1}\right)+\varepsilon \eta_{3}\left(1+\beta_{1} \bar{N}_{1}\right)\right]}{a \varepsilon\left(1+\beta_{1} \bar{N}_{1}\right)\left(1+\beta_{3} \bar{M}_{1}\right)} \mathrm{U} \\
& -\frac{\eta_{3} \bar{W}_{1} M}{1+\beta_{3} \bar{M}_{1}}-\frac{\lambda \bar{W}_{1}\left[b \eta_{1}\left(1+\beta_{3} \bar{M}_{1}\right)+\varepsilon \eta_{3}\left(1+\beta_{1} \bar{N}_{1}\right)\right]}{a \varepsilon\left(1+\beta_{1} \bar{N}_{1}\right)\left(1+\beta_{3} \bar{M}_{1}\right)} \mathrm{u} \frac{\bar{M}_{1}}{M} \\
& +\frac{\bar{W}_{1}\left[b \eta_{1}\left(1+\beta_{3} \bar{M}_{1}\right)+\varepsilon \eta_{3}\left(1+\beta_{1} \bar{N}_{1}\right)\right]}{\varepsilon\left(1+\beta_{1} \bar{N}_{1}\right)\left(1+\beta_{3} \bar{M}_{1}\right)} \bar{M}_{1}+\frac{\mu \bar{W}_{1}\left[b \eta_{1}\left(1+\beta_{3} \bar{M}_{1}\right)+\varepsilon \eta_{3}\left(1+\beta_{1} \bar{N}_{1}\right)\right]}{a \varepsilon\left(1+\beta_{1} \bar{N}_{1}\right)\left(1+\beta_{3} \bar{M}_{1}\right)} \bar{M}_{1} \\
& -\frac{\eta_{1} \bar{W}_{1} N}{1+\beta_{1} \bar{N}_{1}}-\frac{b \eta_{1} \bar{W}_{1} M}{\varepsilon\left(1+\beta_{1} \bar{N}_{1}\right)} \frac{\bar{N}_{1}}{N}+\frac{\eta_{1} \bar{W}_{1} \bar{N}_{1}}{1+\beta_{1} \bar{N}_{1}}-\frac{\mu \pi \bar{W}_{1}\left[b \eta_{1}\left(1+\beta_{3} \bar{M}_{1}\right)+\varepsilon \eta_{3}\left(1+\beta_{1} \bar{N}_{1}\right)\right]}{\sigma a \varepsilon\left(1+\beta_{1} \bar{N}_{1}\right)\left(1+\beta_{3} \bar{M}_{1}\right)} P .
\end{aligned}
$$


Using the equilibrium conditions for $\bar{Ð}_{1}$, we get

In addition,

$$
\begin{aligned}
& \rho=\alpha \bar{W}_{1}+\frac{\eta_{1} \bar{W}_{1} \bar{N}_{1}}{1+\beta_{1} \bar{N}_{1}}+\frac{\eta_{2} \bar{W}_{1} \overline{\mathrm{U}}_{1}}{1+\beta_{2} \overline{\mathrm{U}}_{1}}+\frac{\eta_{3} \bar{W}_{1} \overline{\mathrm{M}}_{1}}{1+\beta_{3} \overline{\mathrm{M}}_{1}}, \\
& \frac{\eta_{1} \bar{W}_{1} \overline{\mathrm{N}}_{1}}{1+\beta_{1} \overline{\mathrm{N}}_{1}}+\frac{\eta_{2} \bar{W}_{1} \overline{\mathrm{U}}_{1}}{1+\beta_{2} \overline{\mathrm{U}}_{1}}+\frac{\eta_{3} \bar{W}_{1} \overline{\mathrm{M}}_{1}}{1+\beta_{3} \overline{\mathrm{M}}_{1}}=(\lambda+\gamma) \overline{\mathrm{U}}_{1}, \\
& \frac{\lambda \overline{\mathrm{U}}_{1}}{\mathrm{a}}=\overline{\mathrm{M}}_{1}, \quad \overline{\mathrm{N}}_{1}=\frac{\mathrm{b} \bar{M}_{1}}{\varepsilon} .
\end{aligned}
$$

$$
\begin{aligned}
\frac{\eta_{1} \bar{W}_{1} \bar{N}_{1}}{1+\beta_{1} \bar{N}_{1}}+\frac{\eta_{3} \bar{W}_{1} \bar{M}_{1}}{1+\beta_{3} \bar{M}_{1}} & =\frac{\bar{W}_{1}\left[\mathrm{~b} \eta_{1}\left(1+\beta_{3} \bar{M}_{1}\right)+\varepsilon \eta_{3}\left(1+\beta_{1} \bar{N}_{1}\right)\right]}{\varepsilon\left(1+\beta_{1} \bar{N}_{1}\right)\left(1+\beta_{3} \bar{M}_{1}\right)} \bar{M}_{1} \\
& =\frac{\lambda \bar{W}_{1}\left[b \eta_{1}\left(1+\beta_{3} \bar{M}_{1}\right)+\varepsilon \eta_{3}\left(1+\beta_{1} \bar{N}_{1}\right)\right]}{a \varepsilon\left(1+\beta_{1} \bar{N}_{1}\right)\left(1+\beta_{3} \bar{M}_{1}\right)} \bar{U}_{1} .
\end{aligned}
$$

Then, we obtain

$$
\begin{aligned}
& \frac{\mathrm{d} \bar{\Theta}_{1}}{\mathrm{dt}}=\left(1-\frac{\bar{W}_{1}}{W}\right)\left(\alpha \bar{W}_{1}-\alpha W\right)+\left(\frac{\eta_{1} \bar{W}_{1} \bar{N}_{1}}{1+\beta_{1} \bar{N}_{1}}+\frac{\eta_{2} \bar{W}_{1} \overline{\mathrm{U}}_{1}}{1+\beta_{2} \overline{\mathrm{U}}_{1}}+\frac{\eta_{3} \bar{W}_{1} \bar{M}_{1}}{1+\beta_{3} \bar{M}_{1}}\right)\left(1-\frac{\bar{W}_{1}}{W}\right) \\
& +\frac{\eta_{1} \bar{W}_{1} \bar{N}_{1}}{1+\beta_{1} \bar{N}_{1}} \frac{N\left(1+\beta_{1} \bar{N}_{1}\right)}{\bar{N}_{1}\left(1+\beta_{1} N\right)}+\frac{\eta_{2} \bar{W}_{1} \bar{u}_{1}}{1+\beta_{2} \bar{U}_{1}} \frac{\mathrm{U}\left(1+\beta_{2} \overline{\mathrm{U}}_{1}\right)}{\overline{\mathrm{U}}_{1}\left(1+\beta_{2} \mathrm{U}\right)}+\frac{\eta_{3} \bar{W}_{1} \bar{M}_{1}}{1+\beta_{3} \bar{M}_{1}} \frac{M\left(1+\beta_{3} \bar{M}_{1}\right)}{\bar{M}_{1}\left(1+\beta_{3} M\right)} \\
& -\frac{\eta_{2} \bar{W}_{1} \overline{\mathrm{U}}_{1}}{1+\beta_{2} \overline{\mathrm{U}}_{1}} \frac{\mathrm{U}}{\overline{\mathrm{U}}_{1}}-\frac{\eta_{1} \overline{\mathrm{W}}_{1} \overline{\mathrm{N}}_{1}}{1+\beta_{1} \overline{\mathrm{N}}_{1}} \frac{W N \overline{\mathrm{U}}_{1}\left(1+\beta_{1} \overline{\mathrm{N}}_{1}\right)}{\bar{W}_{1} \overline{\mathrm{N}}_{1} \mathrm{U}\left(1+\beta_{1} \mathrm{~N}\right)}-\frac{\eta_{2} \bar{W}_{1} \overline{\mathrm{U}}_{1}}{1+\beta_{2} \overline{\mathrm{U}}_{1}} \frac{W\left(1+\beta_{2} \overline{\mathrm{U}}_{1}\right)}{\bar{W}_{1}\left(1+\beta_{2} \mathrm{U}\right)} \\
& -\frac{\eta_{3} \bar{W}_{1} \bar{M}_{1}}{1+\beta_{3} \bar{M}_{1}} \frac{W M \bar{U}_{1}\left(1+\beta_{3} \bar{M}_{1}\right)}{\bar{W}_{1} \bar{M}_{1} \mathrm{U}\left(1+\beta_{3} M\right)}+\frac{\eta_{1} \bar{W}_{1} \bar{N}_{1}}{1+\beta_{1} \bar{N}_{1}}+\frac{\eta_{2} \bar{W}_{1} \overline{\mathrm{U}}_{1}}{1+\beta_{2} \overline{\mathrm{U}}_{1}}+\frac{\eta_{3} \bar{W}_{1} \bar{M}_{1}}{1+\beta_{3} \bar{M}_{1}}-\frac{\eta_{3} \bar{W}_{1} \bar{M}_{1}}{1+\beta_{3} \bar{M}_{1}} \frac{M}{\bar{M}_{1}} \\
& -\frac{\eta_{1} \bar{W}_{1} \bar{N}_{1}}{1+\beta_{1} \bar{N}_{1}} \frac{U \bar{M}_{1}}{\bar{U}_{1} M}-\frac{\eta_{3} \bar{W}_{1} \bar{M}_{1}}{1+\beta_{3} \bar{M}_{1}} \frac{U \bar{M}_{1}}{\bar{u}_{1} M}+\frac{\eta_{1} \bar{W}_{1} \bar{N}_{1}}{1+\beta_{1} \bar{N}_{1}}+\frac{\eta_{3} \bar{W}_{1} \bar{M}_{1}}{1+\beta_{3} \bar{M}_{1}}-\frac{\eta_{1} \bar{W}_{1} \bar{N}_{1}}{1+\beta_{1} \bar{N}_{1}} \frac{N}{\bar{N}_{1}} \\
& -\frac{\eta_{1} \bar{W}_{1} \bar{N}_{1}}{1+\beta_{1} \bar{N}_{1}} \frac{M \bar{N}_{1}}{\bar{M}_{1} \mathrm{~N}}+\frac{\eta_{1} \bar{W}_{1} \bar{N}_{1}}{1+\beta_{1} \bar{N}_{1}}+\frac{\mu \bar{W}_{1}\left[b \eta_{1}\left(1+\beta_{3} \bar{M}_{1}\right)+\varepsilon \eta_{3}\left(1+\beta_{1} \bar{N}_{1}\right)\right]}{a \varepsilon\left(1+\beta_{1} \bar{N}_{1}\right)\left(1+\beta_{3} \bar{M}_{1}\right)}\left(\bar{M}_{1}-\frac{\pi}{\sigma}\right) P \\
& =-\alpha \frac{\left(W-\bar{W}_{1}\right)^{2}}{W}+\frac{\eta_{1} \bar{W}_{1} \bar{N}_{1}}{1+\beta_{1} \bar{N}_{1}}\left[\frac{N\left(1+\beta_{1} \bar{N}_{1}\right)}{\bar{N}_{1}\left(1+\beta_{1} N\right)}-\frac{N}{\bar{N}_{1}}\right]+\frac{\eta_{2} \bar{W}_{1} \bar{U}_{1}}{1+\beta_{2} \bar{U}_{1}}\left[\frac{\mathrm{U}\left(1+\beta_{2} \bar{u}_{1}\right)}{\overline{\mathrm{U}}_{1}\left(1+\beta_{2} \mathrm{U}\right)}-\frac{\mathrm{u}}{\overline{\mathrm{U}}_{1}}\right] \\
& +\frac{\eta_{3} \bar{W}_{1} \bar{M}_{1}}{1+\beta_{3} \bar{M}_{1}}\left[\frac{M\left(1+\beta_{3} \bar{M}_{1}\right)}{\bar{M}_{1}\left(1+\beta_{3} M\right)}-\frac{M}{\bar{M}_{1}}\right]+\frac{\eta_{1} \bar{W}_{1} \bar{N}_{1}}{1+\beta_{1} \bar{N}_{1}}\left[4-\frac{\bar{W}_{1}}{W}-\frac{W N \bar{U}_{1}\left(1+\beta_{1} \bar{N}_{1}\right)}{\bar{W}_{1} \bar{N}_{1} \mathrm{U}\left(1+\beta_{1} N\right)}-\frac{U \bar{M}_{1}}{\bar{U}_{1} M}-\frac{M \bar{N}_{1}}{\bar{M}_{1} N}\right] \\
& +\frac{\eta_{2} \bar{W}_{1} \bar{u}_{1}}{1+\beta_{2} \bar{u}_{1}}\left[2-\frac{\bar{W}_{1}}{W}-\frac{W\left(1+\beta_{2} \bar{u}_{1}\right)}{\bar{W}_{1}\left(1+\beta_{2} \mathrm{u}\right)}\right]+\frac{\eta_{3} \bar{W}_{1} \bar{M}_{1}}{1+\beta_{3} \bar{M}_{1}}\left[3-\frac{\bar{W}_{1}}{W}-\frac{W M \bar{u}_{1}\left(1+\beta_{3} \bar{M}_{1}\right)}{\bar{W}_{1} \bar{M}_{1} \mathrm{U}\left(1+\beta_{3} M\right)}-\frac{u \bar{M}_{1}}{\bar{u}_{1} M}\right] \\
& +\frac{\mu \bar{W}_{1}\left[b \eta_{1}\left(1+\beta_{3} \bar{M}_{1}\right)+\varepsilon \eta_{3}\left(1+\beta_{1} \bar{N}_{1}\right)\right]}{a \varepsilon\left(1+\beta_{1} \bar{N}_{1}\right)\left(1+\beta_{3} \bar{M}_{1}\right)}\left(\bar{M}_{1}-\frac{\pi}{\sigma}\right) P \text {. }
\end{aligned}
$$

Adding and subtracting terms of above equation, we derive

$$
\begin{aligned}
& \frac{\mathrm{d} \bar{\Theta}_{1}}{\mathrm{dt}}=-\alpha \frac{\left(\mathrm{W}-\overline{\mathrm{W}}_{1}\right)^{2}}{\mathrm{~W}}+\Delta_{1} \frac{\eta_{1} \overline{\mathrm{W}}_{1} \overline{\mathrm{N}}_{1}}{1+\beta_{1} \overline{\mathrm{N}}_{1}}+\Delta_{2} \frac{\eta_{2} \overline{\mathrm{W}}_{1} \overline{\mathrm{U}}_{1}}{1+\beta_{2} \overline{\mathrm{U}}_{1}}+\Delta_{3} \frac{\eta_{3} \overline{\mathrm{W}}_{1} \overline{\mathrm{M}}_{1}}{1+\beta_{3} \overline{\mathrm{M}}_{1}} \\
& +\frac{\eta_{1} \bar{W}_{1} \bar{N}_{1}}{1+\beta_{1} \bar{N}_{1}}\left[5-\frac{\bar{W}_{1}}{W}-\frac{W N \bar{U}_{1}\left(1+\beta_{1} \bar{N}_{1}\right)}{\bar{W}_{1} \bar{N}_{1} \mathrm{U}\left(1+\beta_{1} N\right)}-\frac{U \bar{M}_{1}}{\bar{U}_{1} M}-\frac{M \bar{N}_{1}}{\bar{M}_{1} N}-\frac{1+\beta_{1} N}{1+\beta_{1} \bar{N}_{1}}\right] \\
& +\frac{\eta_{2} \bar{W}_{1} \overline{\mathrm{U}}_{1}}{1+\beta_{2} \overline{\mathrm{U}}_{1}}\left[3-\frac{\bar{W}_{1}}{\mathrm{~W}}-\frac{W\left(1+\beta_{2} \overline{\mathrm{U}}_{1}\right)}{\overline{\mathrm{W}}_{1}\left(1+\beta_{2} \mathrm{u}\right)}-\frac{1+\beta_{2} \mathrm{u}}{1+\beta_{2} \overline{\mathrm{U}}_{1}}\right] \\
& +\frac{\eta_{3} \bar{W}_{1} \bar{M}_{1}}{1+\beta_{3} \bar{M}_{1}}\left[4-\frac{\bar{W}_{1}}{W}-\frac{W M \bar{U}_{1}\left(1+\beta_{3} \bar{M}_{1}\right)}{\bar{W}_{1} \bar{M}_{1} \mathrm{U}\left(1+\beta_{3} M\right)}-\frac{U \bar{M}_{1}}{\bar{U}_{1} M}-\frac{1+\beta_{3} M}{1+\beta_{3} \bar{M}_{1}}\right] \\
& +\frac{\mu \bar{W}_{1}\left[b \eta_{1}\left(1+\beta_{3} \bar{M}_{1}\right)+\varepsilon \eta_{3}\left(1+\beta_{1} \bar{N}_{1}\right)\right]}{a \varepsilon\left(1+\beta_{1} \bar{N}_{1}\right)\left(1+\beta_{3} \bar{M}_{1}\right)}\left(\bar{M}_{1}-\frac{\pi}{\sigma}\right) P,
\end{aligned}
$$


where

$$
\begin{aligned}
& \Delta_{1}=\frac{N\left(1+\beta_{1} \bar{N}_{1}\right)}{\bar{N}_{1}\left(1+\beta_{1} N\right)}-\frac{N}{\bar{N}_{1}}-1+\frac{1+\beta_{1} N}{1+\beta_{1} \bar{N}_{1}}=-\frac{\beta_{1}\left(N-\bar{N}_{1}\right)^{2}}{\left(1+\beta_{1} N\right)\left(1+\beta_{1} \bar{N}_{1}\right) \bar{N}_{1}}, \\
& \Delta_{2}=\frac{U\left(1+\beta_{2} \bar{U}_{1}\right)}{\overline{\mathrm{U}}_{1}\left(1+\beta_{2} \mathrm{U}\right)}-\frac{\mathrm{U}}{\overline{\mathrm{U}}_{1}}-1+\frac{1+\beta_{2} \mathrm{U}}{1+\beta_{2} \overline{\mathrm{U}}_{1}}=-\frac{\beta_{2}\left(\mathrm{U}-\overline{\mathrm{U}}_{1}\right)^{2}}{\left(1+\beta_{2} \mathrm{U}\right)\left(1+\beta_{2} \overline{\mathrm{U}}_{1}\right) \overline{\mathrm{U}}_{1}}, \\
& \Delta_{3}=\frac{M\left(1+\beta_{3} \overline{\mathrm{M}}_{1}\right)}{\overline{\mathrm{M}}_{1}\left(1+\beta_{3} \mathrm{M}\right)}-\frac{\mathrm{M}}{\overline{\mathrm{M}}_{1}}-1+\frac{1+\beta_{3} \mathrm{M}}{1+\beta_{3} \overline{\mathrm{M}}_{1}}=-\frac{\beta_{3}\left(\mathrm{M}-\overline{\mathrm{M}}_{1}\right)^{2}}{\left(1+\beta_{3} \mathrm{M}\right)\left(1+\beta_{3} \overline{\mathrm{M}}_{1}\right) \overline{\mathrm{M}}_{1}} .
\end{aligned}
$$

Therefore, Eq. (3.11) becomes

$$
\begin{aligned}
& \frac{d \bar{\Theta}_{1}}{d t}=-\alpha \frac{\left(W-\bar{W}_{1}\right)^{2}}{W}-\frac{\beta_{1} \eta_{1} \bar{W}_{1}\left(N-\bar{N}_{1}\right)^{2}}{\left(1+\beta_{1} N\right)\left(1+\beta_{1} \bar{N}_{1}\right)^{2}} \\
& -\frac{\beta_{2} \eta_{2} \bar{W}_{1}\left(\mathrm{U}-\overline{\mathrm{U}}_{1}\right)^{2}}{\left(1+\beta_{2} \mathrm{U}\right)\left(1+\beta_{2} \overline{\mathrm{U}}_{1}\right)^{2}}-\frac{\beta_{3} \eta_{3} \bar{W}_{1}\left(M-\bar{M}_{1}\right)^{2}}{\left(1+\beta_{3} \mathrm{M}\right)\left(1+\beta_{3} \bar{M}_{1}\right)^{2}} \\
& +\frac{\eta_{1} \bar{W}_{1} \bar{N}_{1}}{1+\beta_{1} \bar{N}_{1}}\left[5-\frac{\bar{W}_{1}}{W}-\frac{W \bar{N}_{1}\left(1+\beta_{1} \bar{N}_{1}\right)}{\bar{W}_{1} \bar{N}_{1} \mathrm{U}\left(1+\beta_{1} \mathrm{~N}\right)}-\frac{\mathrm{U} \bar{M}_{1}}{\bar{U}_{1} M}-\frac{M \bar{N}_{1}}{\bar{M}_{1} N}-\frac{1+\beta_{1} N}{1+\beta_{1} \bar{N}_{1}}\right] \\
& +\frac{\eta_{2} \bar{W}_{1} \overline{\mathrm{U}}_{1}}{1+\beta_{2} \overline{\mathrm{U}}_{1}}\left[3-\frac{\bar{W}_{1}}{\mathrm{~W}}-\frac{W\left(1+\beta_{2} \overline{\mathrm{U}}_{1}\right)}{\overline{\mathrm{W}}_{1}\left(1+\beta_{2} \mathrm{U}\right)}-\frac{1+\beta_{2} \mathrm{U}}{1+\beta_{2} \overline{\mathrm{U}}_{1}}\right] \\
& +\frac{\eta_{3} \bar{W}_{1} \bar{M}_{1}}{1+\beta_{3} \bar{M}_{1}}\left[4-\frac{\bar{W}_{1}}{W}-\frac{W M \bar{U}_{1}\left(1+\beta_{3} \bar{M}_{1}\right)}{\bar{W}_{1} \bar{M}_{1} \mathrm{U}\left(1+\beta_{3} M\right)}-\frac{u \bar{M}_{1}}{\bar{U}_{1} M}-\frac{1+\beta_{3} M}{1+\beta_{3} \bar{M}_{1}}\right] \\
& +\frac{\mu \bar{W}_{1}\left[\operatorname{b\eta }_{1}\left(1+\beta_{3} \bar{M}_{1}\right)+\varepsilon \eta_{3}\left(1+\beta_{1} \bar{N}_{1}\right)\right]}{a \varepsilon\left(1+\beta_{1} \bar{N}_{1}\right)\left(1+\beta_{3} \bar{M}_{1}\right)}\left(\bar{M}_{1}-\bar{M}_{2}\right) P .
\end{aligned}
$$

Since the arithmetical mean is greater than or equal to the geometrical mean, then

$$
\begin{aligned}
& \frac{\bar{W}_{1}}{W}+\frac{W N_{U_{1}}\left(1+\beta_{1} \bar{N}_{1}\right)}{\bar{W}_{1} \bar{N}_{1} \mathrm{U}\left(1+\beta_{1} N\right)}+\frac{U \bar{M}_{1}}{\bar{U}_{1} M}+\frac{M \bar{N}_{1}}{\bar{M}_{1} \mathrm{~N}}+\frac{1+\beta_{1} \mathrm{~N}}{1+\beta_{1} \bar{N}_{1}} \geqslant 5, \\
& \frac{\bar{W}_{1}}{W}+\frac{W\left(1+\beta_{2} \overline{\mathrm{U}}_{1}\right)}{\bar{W}_{1}\left(1+\beta_{2} \mathrm{U}\right)}+\frac{1+\beta_{2} \mathrm{U}}{1+\beta_{2} \overline{\mathrm{U}}_{1}} \geqslant 3, \\
& \frac{\bar{W}_{1}}{W}+\frac{W M \bar{U}_{1}\left(1+\beta_{3} \bar{M}_{1}\right)}{\bar{W}_{1} \bar{M}_{1} \mathrm{U}\left(1+\beta_{3} M\right)}+\frac{u \bar{M}_{1}}{\bar{U}_{1} M}+\frac{1+\beta_{3} M}{1+\beta_{3} \bar{M}_{1}} \geqslant 4 \text {. }
\end{aligned}
$$

Thus, the fifth, sixth and seventh terms of Eq. (3.12) are less than or equal to zero. We note that if $\overline{\mathfrak{R}}_{1} \leqslant 1$, then $\bar{Ð}_{2}$ does not exist since $\overline{\mathrm{P}}_{2}=\frac{\mathrm{a}}{\mu}\left(\overline{\mathfrak{R}}_{1}-1\right) \leqslant 0$. This implies that, $\dot{\mathrm{P}}(\mathrm{t})=\sigma\left(\mathrm{M}-\frac{\pi}{\sigma}\right) \mathrm{P} \leqslant 0$ for all $\mathrm{P}>0$. Thus, $\bar{M}_{1} \leqslant \frac{\pi}{\sigma}=\bar{M}_{2}$. Therefore, $\frac{\mathrm{d} \bar{\Theta}_{1}}{\mathrm{dt}} \leqslant 0$ for all $\mathrm{W}, \mathrm{U}, \mathrm{M}, \mathrm{N}, \mathrm{P}>0$ with equality holding when $W=\bar{W}_{1}, U=\bar{U}_{1}, M=\bar{M}_{1}, N=\bar{N}_{1}$ and $P=0$. Let $\bar{\Upsilon}_{1}^{\prime}$ be the largest invariant subset of $\bar{\Upsilon}_{1}=\left\{(W, U, M, N, P): \frac{d \bar{\Theta}_{1}}{d t}=0\right\}$. It can be seen that $\bar{\Upsilon}_{1}^{\prime}=\left\{\bar{Ð}_{1}\right\}$ and $\bar{Ð}_{1}$ is G.A.S using LaSalle's invariance principle.

Theorem 3.3. For system (3.1), suppose that $\overline{\mathfrak{R}}_{1}>1$, then $\bar{Ð}_{2}$ is G.A.S.

Proof. Define a function $\bar{\Theta}_{2}(\mathrm{~W}, \mathrm{U}, \mathrm{M}, \mathrm{N}, \mathrm{P})$ as:

$$
\bar{\Theta}_{2}=\bar{W}_{2} \digamma\left(\frac{W}{\bar{W}_{2}}\right)+\bar{U}_{2} \digamma\left(\frac{\mathrm{u}}{\overline{\mathrm{U}}_{2}}\right)+\frac{\bar{W}_{2}\left[\mathrm{~b} \eta_{1}\left(1+\beta_{3} \bar{M}_{2}\right)+\varepsilon \eta_{3}\left(1+\beta_{1} \bar{N}_{2}\right)\right]}{\varepsilon\left(\mathrm{a}+\mu \overline{\mathrm{P}}_{2}\right)\left(1+\beta_{1} \overline{\mathrm{N}}_{2}\right)\left(1+\beta_{3} \overline{\mathrm{M}}_{2}\right)} \bar{M}_{2} \digamma\left(\frac{\mathrm{M}}{\overline{\mathrm{M}}_{2}}\right)
$$




$$
+\frac{\eta_{1} \bar{W}_{2}}{\varepsilon\left(1+\beta_{1} \bar{N}_{2}\right)} \bar{N}_{2} \digamma\left(\frac{N}{\bar{N}_{2}}\right)+\frac{\mu \bar{W}_{2}\left[b \eta_{1}\left(1+\beta_{3} \bar{M}_{2}\right)+\varepsilon \eta_{3}\left(1+\beta_{1} \bar{N}_{2}\right)\right]}{\sigma \varepsilon\left(a+\mu \bar{P}_{2}\right)\left(1+\beta_{1} \bar{N}_{2}\right)\left(1+\beta_{3} \bar{M}_{2}\right)} \overline{\mathrm{P}}_{2} \digamma\left(\frac{\mathrm{P}}{\overline{\mathrm{P}}_{2}}\right) .
$$

We calculate $\frac{\mathrm{d} \bar{\Theta}_{2}}{\mathrm{dt}}$ as:

$$
\begin{aligned}
\frac{d \bar{\Theta}_{2}}{d t}= & \left(1-\frac{\bar{W}_{2}}{W}\right)\left(\rho-\alpha W-\frac{\eta_{1} W N}{1+\beta_{1} N}-\frac{\eta_{2} W U}{1+\beta_{2} \mathrm{U}}-\frac{\eta_{3} W M}{1+\beta_{3} M}\right) \\
& +\left(1-\frac{\bar{U}_{2}}{\mathrm{U}}\right)\left(\frac{\eta_{1} W N}{1+\beta_{1} N}+\frac{\eta_{2} W U}{1+\beta_{2} \mathrm{U}}+\frac{\eta_{3} W M}{1+\beta_{3} M}-(\lambda+\gamma) \mathrm{U}\right) \\
& +\frac{\bar{W}_{2}\left[b \eta_{1}\left(1+\beta_{3} \bar{M}_{2}\right)+\varepsilon \eta_{3}\left(1+\beta_{1} \bar{N}_{2}\right)\right]}{\varepsilon\left(a+\mu \bar{P}_{2}\right)\left(1+\beta_{1} \bar{N}_{2}\right)\left(1+\beta_{3} \bar{M}_{2}\right)}\left(1-\frac{\bar{M}_{2}}{M}\right)(\lambda \mathrm{U}-a M-\mu P M) \\
& +\frac{\eta_{1} \bar{W}_{2}}{\varepsilon\left(1+\beta_{1} \bar{N}_{2}\right)}\left(1-\frac{\bar{N}_{2}}{N}\right)(b M-\varepsilon N) \\
& +\frac{\mu \bar{W}_{2}\left[b \eta_{1}\left(1+\beta_{3} \bar{M}_{2}\right)+\varepsilon \eta_{3}\left(1+\beta_{1} \bar{N}_{2}\right)\right]}{\sigma \varepsilon\left(a+\mu \bar{P}_{2}\right)\left(1+\beta_{1} \bar{N}_{2}\right)\left(1+\beta_{3} \bar{M}_{2}\right)}\left(1-\frac{\bar{P}_{2}}{P}\right)(\sigma P M-\pi P) .
\end{aligned}
$$

Collecting terms of Eq. (3.13), we derive

$$
\begin{aligned}
& \frac{d \bar{\Theta}_{2}}{d t}=\left(1-\frac{\bar{W}_{2}}{W}\right)(\rho-\alpha W)+\frac{\eta_{1} \bar{W}_{2} N}{1+\beta_{1} N}+\frac{\eta_{2} \bar{W}_{2} U}{1+\beta_{2} \mathrm{U}}+\frac{\eta_{3} \bar{W}_{2} M}{1+\beta_{3} M}-(\lambda+\gamma) U-\frac{\eta_{1} W N}{1+\beta_{1} N} \frac{\bar{U}_{2}}{U} \\
& -\frac{\eta_{2} W U}{1+\beta_{2} \mathrm{U}} \frac{\overline{\mathrm{U}}_{2}}{\mathrm{U}}-\frac{\eta_{3} W M}{1+\beta_{3} M} \frac{\overline{\mathrm{U}}_{2}}{\mathrm{U}}+(\lambda+\gamma) \overline{\mathrm{U}}_{2}+\frac{\lambda \bar{W}_{2}\left[\mathrm{~b} \eta_{1}\left(1+\beta_{3} \bar{M}_{2}\right)+\varepsilon \eta_{3}\left(1+\beta_{1} \bar{N}_{2}\right)\right]}{\varepsilon\left(a+\mu \bar{P}_{2}\right)\left(1+\beta_{1} \bar{N}_{2}\right)\left(1+\beta_{3} \bar{M}_{2}\right)} \mathrm{U} \\
& -\frac{a \bar{W}_{2}\left[b \eta_{1}\left(1+\beta_{3} \bar{M}_{2}\right)+\varepsilon \eta_{3}\left(1+\beta_{1} \bar{N}_{2}\right)\right]}{\varepsilon\left(a+\mu \bar{P}_{2}\right)\left(1+\beta_{1} \bar{N}_{2}\right)\left(1+\beta_{3} \bar{M}_{2}\right)} M-\frac{\lambda \bar{W}_{2}\left[b \eta_{1}\left(1+\beta_{3} \bar{M}_{2}\right)+\varepsilon \eta_{3}\left(1+\beta_{1} \bar{N}_{2}\right)\right]}{\varepsilon\left(a+\mu \bar{P}_{2}\right)\left(1+\beta_{1} \bar{N}_{2}\right)\left(1+\beta_{3} \bar{M}_{2}\right)} u \frac{\bar{M}_{2}}{M} \\
& +\frac{a \bar{W}_{2}\left[b \eta_{1}\left(1+\beta_{3} \bar{M}_{2}\right)+\varepsilon \eta_{3}\left(1+\beta_{1} \bar{N}_{2}\right)\right]}{\varepsilon\left(a+\mu \bar{P}_{2}\right)\left(1+\beta_{1} \bar{N}_{2}\right)\left(1+\beta_{3} \bar{M}_{2}\right)} \bar{M}_{2}+\frac{\mu \bar{W}_{2}\left[b \eta_{1}\left(1+\beta_{3} \bar{M}_{2}\right)+\varepsilon \eta_{3}\left(1+\beta_{1} \bar{N}_{2}\right)\right]}{\varepsilon\left(a+\mu \bar{P}_{2}\right)\left(1+\beta_{1} \bar{N}_{2}\right)\left(1+\beta_{3} \bar{M}_{2}\right)} P \bar{M}_{2} \\
& +\frac{b \eta_{1} \bar{W}_{2} M}{\varepsilon\left(1+\beta_{1} \bar{N}_{2}\right)}-\frac{\eta_{1} \bar{W}_{2} N}{1+\beta_{1} \bar{N}_{2}}-\frac{b \eta_{1} \bar{W}_{2} M}{\varepsilon\left(1+\beta_{1} \bar{N}_{2}\right)} \frac{\bar{N}_{2}}{N}+\frac{\eta_{1} \bar{W}_{2} \bar{N}_{2}}{1+\beta_{1} \bar{N}_{2}} \\
& -\frac{\mu \pi \bar{W}_{2}\left[b \eta_{1}\left(1+\beta_{3} \bar{M}_{2}\right)+\varepsilon \eta_{3}\left(1+\beta_{1} \bar{N}_{2}\right)\right]}{\sigma \varepsilon\left(a+\mu \bar{P}_{2}\right)\left(1+\beta_{1} \bar{N}_{2}\right)\left(1+\beta_{3} \bar{M}_{2}\right)} P-\frac{\mu \bar{W}_{2}\left[b \eta_{1}\left(1+\beta_{3} \bar{M}_{2}\right)+\varepsilon \eta_{3}\left(1+\beta_{1} \bar{N}_{2}\right)\right]}{\varepsilon\left(a+\mu \bar{P}_{2}\right)\left(1+\beta_{1} \bar{N}_{2}\right)\left(1+\beta_{3} \bar{M}_{2}\right)} \bar{P}_{2} M \\
& +\frac{\mu \pi \bar{W}_{2}\left[b \eta_{1}\left(1+\beta_{3} \bar{M}_{2}\right)+\varepsilon \eta_{3}\left(1+\beta_{1} \bar{N}_{2}\right)\right]}{\sigma \varepsilon\left(a+\mu \bar{P}_{2}\right)\left(1+\beta_{1} \bar{N}_{2}\right)\left(1+\beta_{3} \bar{M}_{2}\right)} \bar{P}_{2} \text {. }
\end{aligned}
$$

Using the equilibrium conditions for $\bar{Ð}_{2}$ :

$$
\begin{aligned}
& \rho=\alpha \bar{W}_{2}+\frac{\eta_{1} \bar{W}_{2} \bar{N}_{2}}{1+\beta_{1} \bar{N}_{2}}+\frac{\eta_{2} \bar{W}_{2} \overline{\mathrm{U}}_{2}}{1+\beta_{2} \overline{\mathrm{U}}_{2}}+\frac{\eta_{3} \bar{W}_{2} \bar{M}_{2}}{1+\beta_{3} \bar{M}_{2}}, \\
& \frac{\eta_{1} \bar{W}_{2} \bar{N}_{2}}{1+\beta_{1} \bar{N}_{2}}+\frac{\eta_{2} \bar{W}_{2} \overline{\mathrm{U}}_{2}}{1+\beta_{2} \overline{\mathrm{U}}_{2}}+\frac{\eta_{3} \bar{W}_{2} \bar{M}_{2}}{1+\beta_{3} \bar{M}_{2}}=(\lambda+\gamma) \overline{\mathrm{U}}_{2}, \\
& \lambda \overline{\mathrm{U}}_{2}=\left(\mathrm{a}+\mu \overline{\mathrm{P}}_{2}\right) \overline{\mathrm{M}}_{2}, \quad \bar{M}_{2}=\frac{\pi}{\sigma}, \quad \overline{\mathrm{N}}_{2}=\frac{b}{\varepsilon} \bar{M}_{2} .
\end{aligned}
$$

Further,

$$
\begin{aligned}
\frac{\eta_{1} \bar{W}_{2} \bar{N}_{2}}{1+\beta_{1} \bar{N}_{2}}+\frac{\eta_{3} \bar{W}_{2} \bar{M}_{2}}{1+\beta_{3} \bar{M}_{2}} & =\frac{\bar{W}_{2}\left[b \eta_{1}\left(1+\beta_{3} \bar{M}_{2}\right)+\varepsilon \eta_{3}\left(1+\beta_{1} \bar{N}_{2}\right)\right]}{\varepsilon\left(1+\beta_{1} \bar{N}_{2}\right)\left(1+\beta_{3} \bar{M}_{2}\right)} \bar{M}_{2} \\
& =\frac{\lambda \bar{W}_{2}\left[b \eta_{1}\left(1+\beta_{3} \bar{M}_{2}\right)+\varepsilon \eta_{3}\left(1+\beta_{1} \bar{N}_{2}\right)\right]}{\varepsilon\left(a+\mu \bar{P}_{2}\right)\left(1+\beta_{1} \bar{N}_{2}\right)\left(1+\beta_{3} \bar{M}_{2}\right)} \bar{U}_{2} .
\end{aligned}
$$


Therefore, we obtain

$$
\begin{aligned}
& \frac{d \bar{\Theta}_{2}}{d t}=\left(1-\frac{\bar{W}_{2}}{W}\right)\left(\alpha \bar{W}_{2}-\alpha W\right)+\left(\frac{\eta_{1} \bar{W}_{2} \bar{N}_{2}}{1+\beta_{1} \bar{N}_{2}}+\frac{\eta_{2} \bar{W}_{2} \bar{U}_{2}}{1+\beta_{2} \overline{\mathrm{U}}_{2}}+\frac{\eta_{3} \bar{W}_{2} \bar{M}_{2}}{1+\beta_{3} \bar{M}_{2}}\right)\left(1-\frac{\bar{W}_{2}}{W}\right) \\
& +\frac{\eta_{1} \bar{W}_{2} \bar{N}_{2}}{1+\beta_{1} \bar{N}_{2}} \frac{N\left(1+\beta_{1} \bar{N}_{2}\right)}{\bar{N}_{2}\left(1+\beta_{1} N\right)}+\frac{\eta_{2} \bar{W}_{2} \overline{\mathrm{U}}_{2}}{1+\beta_{2} \overline{\mathrm{U}}_{2}} \frac{\mathrm{U}\left(1+\beta_{2} \overline{\mathrm{U}}_{2}\right)}{\overline{\mathrm{U}}_{2}\left(1+\beta_{2} \mathrm{U}\right)}+\frac{\eta_{3} \overline{\mathrm{W}}_{2} \overline{\mathrm{M}}_{2}}{1+\beta_{3} \overline{\mathrm{M}}_{2}} \frac{\mathrm{M}\left(1+\beta_{3} \overline{\mathrm{M}}_{2}\right)}{\overline{\mathrm{M}}_{2}\left(1+\beta_{3} \mathrm{M}\right)} \\
& -\frac{\eta_{2} \bar{W}_{2} \overline{\mathrm{U}}_{2}}{1+\beta_{2} \overline{\mathrm{U}}_{2}} \frac{\mathrm{U}}{\overline{\mathrm{U}}_{2}}-\frac{\eta_{1} \bar{W}_{2} \overline{\mathrm{N}}_{2}}{1+\beta_{1} \overline{\mathrm{N}}_{2}} \frac{W N \overline{\mathrm{U}}_{2}\left(1+\beta_{1} \overline{\mathrm{N}}_{2}\right)}{\bar{W}_{2} \overline{\mathrm{N}}_{2} \mathrm{U}\left(1+\beta_{1} \mathrm{~N}\right)}-\frac{\eta_{2} \bar{W}_{2} \overline{\mathrm{U}}_{2}}{1+\beta_{2} \overline{\mathrm{U}}_{2}} \frac{W\left(1+\beta_{2} \overline{\mathrm{U}}_{2}\right)}{\bar{W}_{2}\left(1+\beta_{2} \mathrm{U}\right)} \\
& -\frac{\eta_{3} \bar{W}_{2} \bar{M}_{2}}{1+\beta_{3} \bar{M}_{2}} \frac{W M \bar{U}_{2}\left(1+\beta_{3} \bar{M}_{2}\right)}{\bar{W}_{2} \bar{M}_{2} \mathrm{U}\left(1+\beta_{3} M\right)}+\frac{\eta_{1} \bar{W}_{2} \bar{N}_{2}}{1+\beta_{1} \bar{N}_{2}}+\frac{\eta_{2} \bar{W}_{2} \overline{\mathrm{U}}_{2}}{1+\beta_{2} \overline{\mathrm{U}}_{2}}+\frac{\eta_{3} \bar{W}_{2} \bar{M}_{2}}{1+\beta_{3} \bar{M}_{2}}-\frac{\eta_{3} \bar{W}_{2} \bar{M}_{2}}{1+\beta_{3} \bar{M}_{2}} \frac{M}{\bar{M}_{2}} \\
& -\frac{\eta_{1} \bar{W}_{2} \bar{N}_{2}}{1+\beta_{1} \bar{N}_{2}} \frac{\mathrm{U} \bar{M}_{2}}{\overline{\mathrm{U}}_{2} M}-\frac{\eta_{3} \bar{W}_{2} \bar{M}_{2}}{1+\beta_{3} \bar{M}_{2}} \frac{\mathrm{U} \bar{M}_{2}}{\overline{\mathrm{U}}_{2} \mathrm{M}}+\frac{\eta_{1} \bar{W}_{2} \bar{N}_{2}}{1+\beta_{1} \overline{\mathrm{N}}_{2}}+\frac{\eta_{3} \bar{W}_{2} \bar{M}_{2}}{1+\beta_{3} \bar{M}_{2}}-\frac{\eta_{1} \bar{W}_{2} \bar{N}_{2}}{1+\beta_{1} \bar{N}_{2}} \frac{\mathrm{N}}{\overline{\mathrm{N}}_{2}} \\
& -\frac{\eta_{1} \bar{W}_{2} \bar{N}_{2}}{1+\beta_{1} \bar{N}_{2}} \frac{M \bar{N}_{2}}{\bar{M}_{2} \mathrm{~N}}+\frac{\eta_{1} \bar{W}_{2} \overline{\mathrm{N}}_{2}}{1+\beta_{1} \bar{N}_{2}} \\
& =-\alpha \frac{\left(W-\bar{W}_{2}\right)^{2}}{W}+\frac{\eta_{1} \bar{W}_{2} \bar{N}_{2}}{1+\beta_{1} \bar{N}_{2}}\left[\frac{N\left(1+\beta_{1} \bar{N}_{2}\right)}{\bar{N}_{2}\left(1+\beta_{1} N\right)}-\frac{N}{\bar{N}_{2}}\right]+\frac{\eta_{2} \bar{W}_{2} \bar{U}_{2}}{1+\beta_{2} \bar{U}_{2}}\left[\frac{\mathrm{U}\left(1+\beta_{2} \bar{U}_{2}\right)}{\bar{U}_{2}\left(1+\beta_{2} \mathrm{U}\right)}-\frac{\mathrm{U}}{\overline{\mathrm{U}}_{2}}\right] \\
& +\frac{\eta_{3} \bar{W}_{2} \bar{M}_{2}}{1+\beta_{3} \bar{M}_{2}}\left[\frac{M\left(1+\beta_{3} \bar{M}_{2}\right)}{\bar{M}_{2}\left(1+\beta_{3} M\right)}-\frac{M}{\bar{M}_{2}}\right]+\frac{\eta_{1} \bar{W}_{2} \bar{N}_{2}}{1+\beta_{1} \bar{N}_{2}}\left[4-\frac{\bar{W}_{2}}{W}-\frac{W N \bar{U}_{2}\left(1+\beta_{1} \bar{N}_{2}\right)}{\bar{W}_{2} \bar{N}_{2} U\left(1+\beta_{1} N\right)}-\frac{U \bar{M}_{2}}{\bar{U}_{2} M}-\frac{M \bar{N}_{2}}{\bar{M}_{2} N}\right] \\
& +\frac{\eta_{2} \bar{W}_{2} \overline{\mathrm{U}}_{2}}{1+\beta_{2} \overline{\mathrm{U}}_{2}}\left[2-\frac{\bar{W}_{2}}{W}-\frac{W\left(1+\beta_{2} \overline{\mathrm{U}}_{2}\right)}{\bar{W}_{2}\left(1+\beta_{2} \mathrm{U}\right)}\right]+\frac{\eta_{3} \bar{W}_{2} \bar{M}_{2}}{1+\beta_{3} \bar{M}_{2}}\left[3-\frac{\bar{W}_{2}}{W}-\frac{W \bar{M}_{2}\left(1+\beta_{3} \bar{M}_{2}\right)}{\bar{W}_{2} \bar{M}_{2} \mathrm{U}\left(1+\beta_{3} M\right)}-\frac{u \bar{M}_{2}}{\overline{\mathrm{U}}_{2} M}\right] .
\end{aligned}
$$

Adding and subtracting terms of above equation, we derive

$$
\begin{aligned}
& \frac{d \bar{\Theta}_{2}}{d t}=-\alpha \frac{\left(W-\bar{W}_{1}\right)^{2}}{W}+\Delta_{4} \frac{\eta_{1} \bar{W}_{2} \bar{N}_{2}}{1+\beta_{1} \bar{N}_{2}}+\Delta_{5} \frac{\eta_{2} \bar{W}_{2} \overline{\mathrm{U}}_{2}}{1+\beta_{2} \overline{\mathrm{U}}_{2}}+\Delta_{6} \frac{\eta_{3} \bar{W}_{2} \bar{M}_{2}}{1+\beta_{3} \bar{M}_{2}} \\
& +\frac{\eta_{1} \bar{W}_{2} \bar{N}_{2}}{1+\beta_{1} \bar{N}_{2}}\left[5-\frac{\bar{W}_{2}}{W}-\frac{W N \bar{U}_{2}\left(1+\beta_{1} \bar{N}_{2}\right)}{\bar{W}_{2} \bar{N}_{2} \mathrm{U}\left(1+\beta_{1} N\right)}-\frac{U \bar{M}_{2}}{\bar{U}_{2} M}-\frac{M \bar{N}_{2}}{\bar{M}_{2} N}-\frac{1+\beta_{1} N}{1+\beta_{1} \bar{N}_{2}}\right] \\
& +\frac{\eta_{2} \bar{W}_{2} \overline{\mathrm{U}}_{2}}{1+\beta_{2} \overline{\mathrm{U}}_{2}}\left[3-\frac{\bar{W}_{2}}{\mathrm{~W}}-\frac{W\left(1+\beta_{2} \overline{\mathrm{U}}_{2}\right)}{\overline{\mathrm{W}}_{2}\left(1+\beta_{2} \mathrm{U}\right)}-\frac{1+\beta_{2} \mathrm{U}}{1+\beta_{2} \overline{\mathrm{U}}_{2}}\right] \\
& +\frac{\eta_{3} \bar{W}_{2} \bar{M}_{2}}{1+\beta_{3} \bar{M}_{2}}\left[4-\frac{\bar{W}_{2}}{W}-\frac{W M \bar{U}_{2}\left(1+\beta_{3} \bar{M}_{2}\right)}{\bar{W}_{2} \bar{M}_{2} \mathrm{U}\left(1+\beta_{3} M\right)}-\frac{u \bar{M}_{2}}{\bar{U}_{2} M}-\frac{1+\beta_{3} M}{1+\beta_{3} \bar{M}_{2}}\right] \text {, }
\end{aligned}
$$

where

$$
\begin{aligned}
& \Delta_{4}=\frac{N\left(1+\beta_{1} \bar{N}_{2}\right)}{\bar{N}_{2}\left(1+\beta_{1} N\right)}-\frac{N}{\bar{N}_{2}}-1+\frac{1+\beta_{1} N}{1+\beta_{1} \bar{N}_{2}}=-\frac{\beta_{1}\left(N-\bar{N}_{2}\right)^{2}}{\left(1+\beta_{1} N\right)\left(1+\beta_{1} \bar{N}_{2}\right) \bar{N}_{2}}, \\
& \Delta_{5}=\frac{U\left(1+\beta_{2} \bar{U}_{2}\right)}{\bar{U}_{2}\left(1+\beta_{2} \mathrm{U}\right)}-\frac{U}{\bar{U}_{2}}-1+\frac{1+\beta_{2} \mathrm{U}}{1+\beta_{2} \overline{\mathrm{U}}_{2}}=-\frac{\beta_{2}\left(\mathrm{U}-\overline{\mathrm{U}}_{2}\right)^{2}}{\left(1+\beta_{2} \mathrm{U}\right)\left(1+\beta_{2} \overline{\mathrm{U}}_{2}\right) \overline{\mathrm{U}}_{2}}, \\
& \Delta_{6}=\frac{\mathrm{M}\left(1+\beta_{3} \bar{M}_{2}\right)}{\bar{M}_{2}\left(1+\beta_{3} M\right)}-\frac{M}{\bar{M}_{2}}-1+\frac{1+\beta_{3} \mathrm{M}}{1+\beta_{3} \bar{M}_{2}}=-\frac{\beta_{3}\left(M-\bar{M}_{2}\right)^{2}}{\left(1+\beta_{3} M\right)\left(1+\beta_{3} \bar{M}_{2}\right) \bar{M}_{2}} .
\end{aligned}
$$

Therefore, Eq. (3.14) becomes

$$
\begin{aligned}
\frac{d \bar{\Theta}_{2}}{d t}= & -\alpha \frac{\left(W-\bar{W}_{2}\right)^{2}}{W}-\frac{\beta_{1} \eta_{1} \bar{W}_{2}\left(N-\bar{N}_{2}\right)^{2}}{\left(1+\beta_{1} N\right)\left(1+\beta_{1} \bar{N}_{2}\right)^{2}}-\frac{\beta_{2} \eta_{2} \bar{W}_{2}\left(U-\bar{U}_{2}\right)^{2}}{\left(1+\beta_{2} \mathrm{U}\right)\left(1+\beta_{2} \bar{U}_{2}\right)^{2}}-\frac{\beta_{3} \eta_{3} \bar{W}_{2}\left(M-\bar{M}_{2}\right)^{2}}{\left(1+\beta_{3} M\right)\left(1+\beta_{3} \bar{M}_{2}\right)^{2}} \\
& +\frac{\eta_{1} \bar{W}_{2} \bar{N}_{2}}{1+\beta_{1} \bar{N}_{2}}\left[5-\frac{\bar{W}_{2}}{W}-\frac{W N \bar{U}_{2}\left(1+\beta_{1} \bar{N}_{2}\right)}{\bar{W}_{2} \bar{N}_{2} \mathrm{U}\left(1+\beta_{1} N\right)}-\frac{U \bar{M}_{2}}{\bar{U}_{2} M}-\frac{M \bar{N}_{2}}{\bar{M}_{2} \mathrm{~N}}-\frac{1+\beta_{1} N}{1+\beta_{1} \bar{N}_{2}}\right]
\end{aligned}
$$




$$
\begin{aligned}
& +\frac{\eta_{2} \bar{W}_{2} \overline{\mathrm{U}}_{2}}{1+\beta_{2} \overline{\mathrm{U}}_{2}}\left[3-\frac{\bar{W}_{2}}{\mathrm{~W}}-\frac{W\left(1+\beta_{2} \overline{\mathrm{U}}_{2}\right)}{\bar{W}_{2}\left(1+\beta_{2} \mathrm{U}\right)}-\frac{1+\beta_{2} \mathrm{U}}{1+\beta_{2} \overline{\mathrm{U}}_{2}}\right] \\
& +\frac{\eta_{3} \bar{W}_{2} \overline{\mathrm{M}}_{2}}{1+\beta_{3} \bar{M}_{2}}\left[4-\frac{\bar{W}_{2}}{W}-\frac{W M \bar{U}_{2}\left(1+\beta_{3} \bar{M}_{2}\right)}{\bar{W}_{2} \bar{M}_{2} \mathrm{U}\left(1+\beta_{3} M\right)}-\frac{u \bar{M}_{2}}{\bar{U}_{2} M}-\frac{1+\beta_{3} M}{1+\beta_{3} \bar{M}_{2}}\right] .
\end{aligned}
$$

In the same way of the proof of Theorem 2.5 , we get that $\bar{Ð}_{2}$ is G.A.S.

\section{Numerical simulations}

In this section, we perform some numerical simulations to clarify the results of Theorems 2.2-2.5. We investigate the effects of CTC transmission and saturation on the dynamics.

\subsection{Stability of the equilibria}

In this subsection, we choose three different initial conditions for model (2.1) as follows:

IV-1: $(\mathrm{W}(0), \mathrm{U}(0), \mathrm{M}(0), \mathrm{N}(0), \mathrm{P}(0))=(600,8,1,3,2)$;

IV-2: $(\mathrm{W}(0), \mathrm{U}(0), \mathrm{M}(0), \mathrm{N}(0), \mathrm{P}(0))=(450,12,2,5,4)$;

IV-3: $(\mathrm{W}(0), \mathrm{U}(0), \mathrm{M}(0), \mathrm{N}(0), \mathrm{P}(0))=(350,20,3,6,6)$.

We consider the following parameters: $\rho=10, \alpha=0.01, a=0.5, \gamma=0.2, \lambda=0.2, b=5, \pi=0.1, \mu=0.2$ and $\varepsilon=2$. Choosing selected values of $\eta_{1}, \eta_{2}, \eta_{3}$ and $\sigma$ under the above initial conditions leads to the following cases.

Stability of $\bigoplus_{0} . \eta_{1}=0.00005, \eta_{2}=0.00006, \eta_{3}=0.00007$ and $\sigma=0.01$. For this set of parameters, we have $\mathfrak{R}_{0}=0.35<1$. Figure 1 illustrates that the solution trajectories initiating with IV-1, IV2 and IV3 reach the equilibrium $\bigoplus_{0}=(1000,0,0,0,0)$. This ensures that $\bigoplus_{0}$ is G.A.S according to the outcome of Theorem 2.2. As a result, the HIV particles can be cleared.

Stability of $\bigoplus_{1} \cdot \eta_{1}=0.0005, \eta_{2}=0.0006, \eta_{3}=0.0007$ and $\sigma=0.1$. With such choice we get $\mathfrak{R}_{1}=0.76<$ $1<3.45=\mathfrak{R}_{0}$. It is clear that, the equilibrium point $\bigoplus_{1}$ exists with $\bigoplus_{1}=(289.86,17.75,7.10,17.75,0)$. Figure 1 shows that the solution trajectories initiating with IV-1, IV2 and IV3 tend to $\bigoplus_{1}$ and this validates Theorem 2.4. This case represents the persistence of the HIV infection but with unstimulated CTLmediated immune response.

Stability of $\bigoplus_{2} . \eta_{1}=0.0005, \eta_{2}=0.0006, \eta_{3}=0.0007$ and $\sigma=0.01$. Then, we calculate $\Re_{0}=3.45>1$ and $\mathfrak{R}_{1}=3.76>1$. The numerical results plotted in Figure 1 show that $\bigoplus_{2}=(523.72,11.91,1.00,2.50,9.41)$ is G.A.S. In this case, a chronic HIV infection with active CTL-mediated is reached.

\subsection{Effect of CTC transmission}

In this subsection, we investigate the influence of different modes of transmission on the HIV dynamics (2.1). We select the parameter $\sigma=0.03$ with IV-1 and study the following cases.

Case (1): HIV dynamics with VTC, silent HIV-infected CTC and active HIV-infected CTC transmissions: In this case, we choose the parameters $\eta_{1}=0.0005, \eta_{2}=0.0006$ and $\eta_{3}=0.0007$. We can see from Figure 2 that the trajectories of the system tend to the equilibrium $\bigoplus_{2}=(389.82,15.25,3.33,8.33,2.08)$.

Case (2): HIV dynamics with both VTC and active HIV-infected CTC transmissions: In this case, we select the values $\eta_{1}=0.0005, \eta_{2}=0$ and $\eta_{3}=0.0007$. From Figure 2, we observe that the solution trajectories converge to the equilibrium $\bigoplus_{2}=(606.06,9.85,3.33,8.33,0.45)$.

Case (3): HIV dynamics with only VTC transmission: Here we consider the parameters $\eta_{1}=0.0005$ and $\eta_{2}=\eta_{3}=0$. Figure 2 shows that the solutions of the system approach the equilibrium $\bigoplus_{1}=(800,5,2,5,0)$.

From the above observations we note that the presence of silent HIV-infected CTC and/or active HIVinfected CTC transmissions increases the infection rate. As a result, the concentration of the susceptible cells is decreased while the concentrations of silent/active HIV-infected cells, free HIV particles and HIVspecific CTLs are increased. 


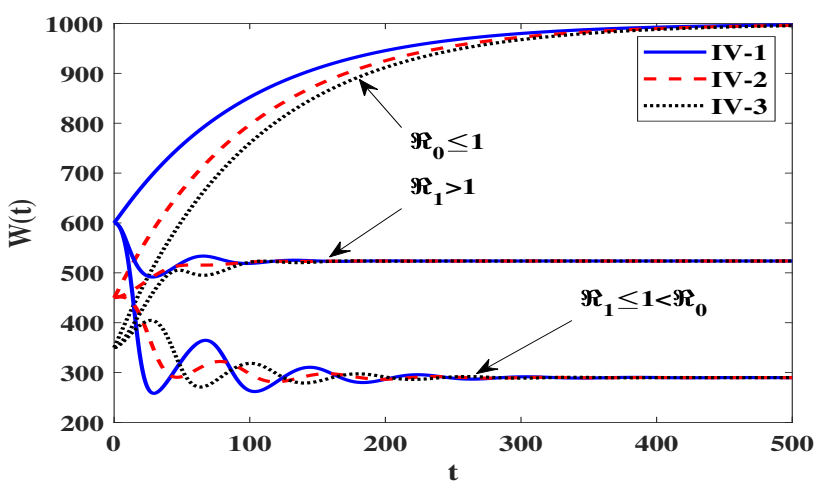

(a) Susceptible $\mathrm{CD} 4^{+} \mathrm{T}$ cells

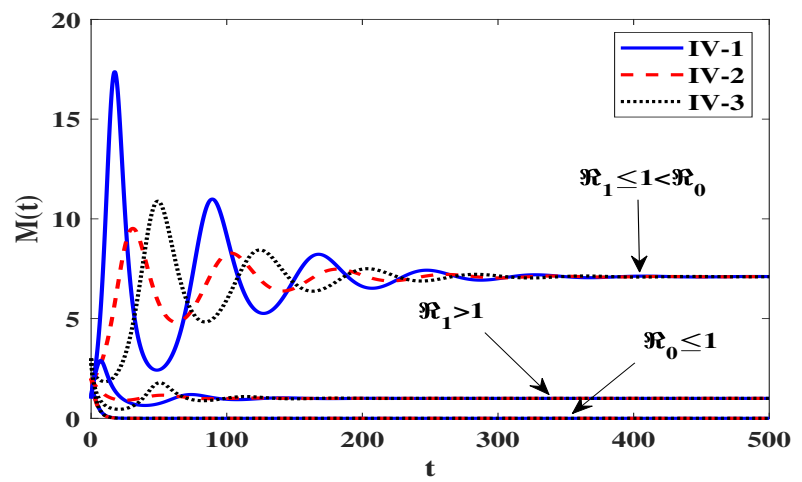

(c) Active HIV-infected cells

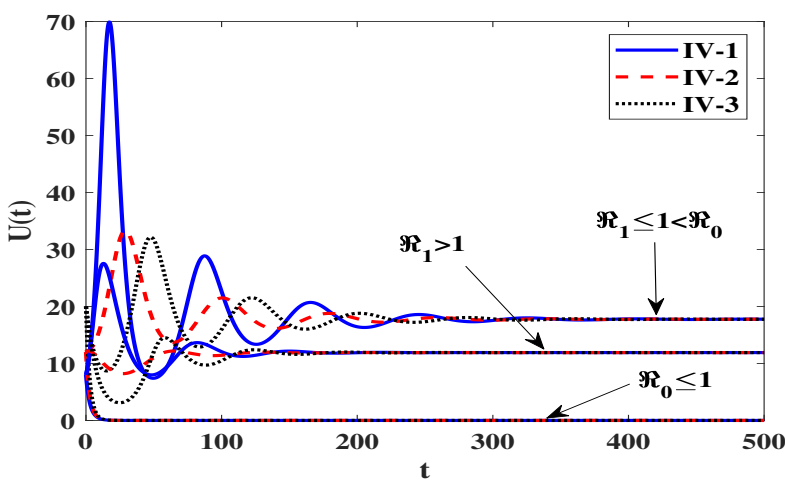

(b) Silent HIV-infected cells

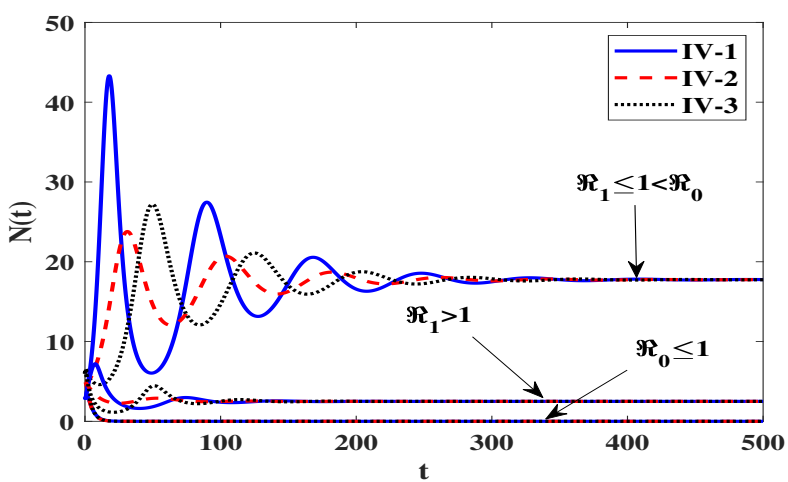

(d) Free HIV particles

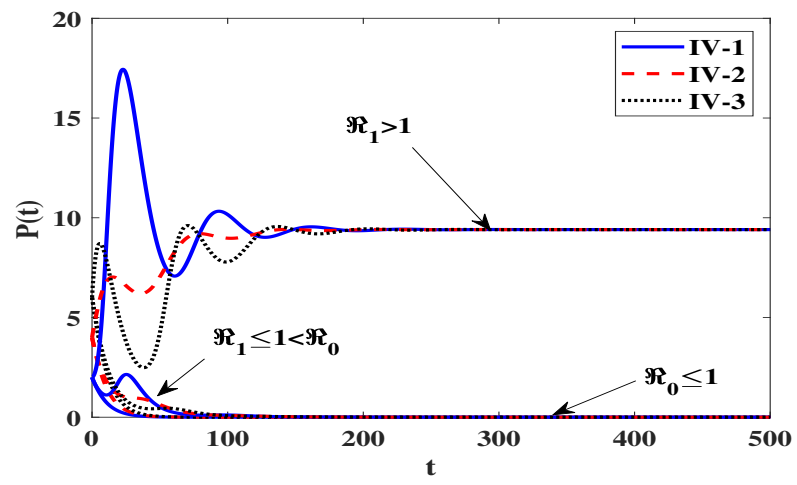

(e) HIV-specific CTLs

Figure 1: The behavior of solution trajectories of system (2.1).

\subsection{Effect of saturation infection}

In this subsection, we study the effect of different values of saturation parameters on the HIV dynamics (3.1). We take the values $\rho=10, \alpha=0.01, \eta_{1}=0.0005, \eta_{2}=0.0006, \eta_{3}=0.0007, a=0.5, \gamma=0.2, \lambda=0.2$, $\mathrm{b}=5, \pi=0.1, \mu=0.2, \varepsilon=2, \sigma=0.05$; with initial condition:

IV-4 : $(\mathrm{W}(0), \mathrm{U}(0), \mathrm{M}(0), \mathrm{N}(0), \mathrm{P}(0))=(600,10,2,5,2)$.

We investigate the following scenarios:

Set 1. $\beta_{1}=\beta_{2}=\beta_{3}=0, \bar{\Re}_{1}=2.73943, \bar{Ð}_{2}=(452.12,13.70,2 ., 5 ., 4.35)$;

Set 2. $\beta_{1}=0.01, \beta_{2}=0.02, \beta_{3}=0.03, \overline{\mathfrak{R}}_{1}=2.44956, \bar{Ð}_{2}=(510.09,12.25,2 ., 5 ., 3.62)$;

Set 3. $\beta_{1}=0.1, \beta_{2}=0.2, \beta_{3}=0.3, \overline{\mathfrak{R}}_{1}=1.51545, \bar{Ð}_{2}=(696.91,7.58,2 ., 5 ., 1.29)$; 
Set 4. $\beta_{1}=1, \beta_{2}=2, \beta_{3}=3, \bar{\Re}_{1}=0.394238, \bar{Ð}_{1}=(935.25,1.62,0.65,1.62,0)$;

Set 5. $\beta_{1}=10, \beta_{2}=20, \beta_{3}=30, \overline{\mathfrak{R}}_{1}=0.047932, \bar{Ð}_{1}=(992.93,0.18,0.07,0.18,0)$.

We can see that the basic HIV reproduction number has a fixed value $\overline{\mathfrak{R}}_{0}=3.45$ in all above scenarios which indicates to the fact that the saturation parameters has no effect on the stability of equilibria. However, Figure 3 illustrates the influence of $\beta_{i}, i=1,2,3$ on the solution trajectories of system (3.1). We observe that as $\beta_{i}$ is increased, the concentration of susceptible CD4 ${ }^{+} \mathrm{T}$ cells is increased while the concentrations of silent/active HIV-infected cells, free HIV particles and HIV-specific CTLs are decreased as illustrated in Figure 3.

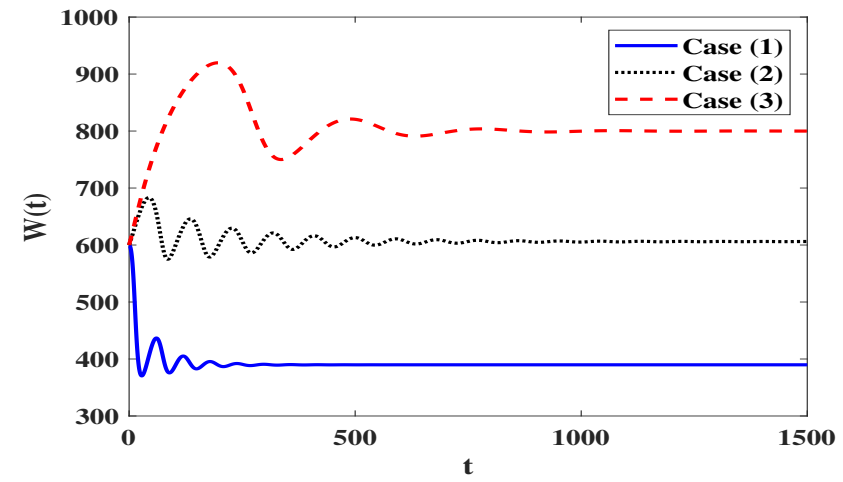

(a) Susceptible $\mathrm{CD} 4^{+} \mathrm{T}$ cells

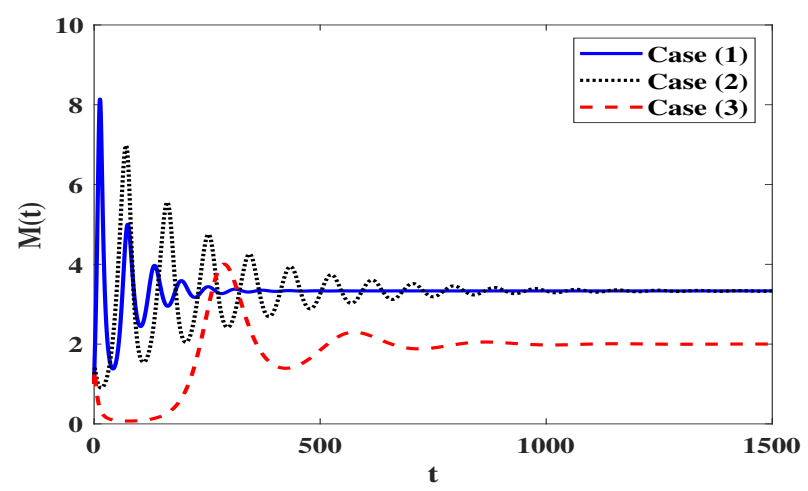

(c) Active HIV-infected cells

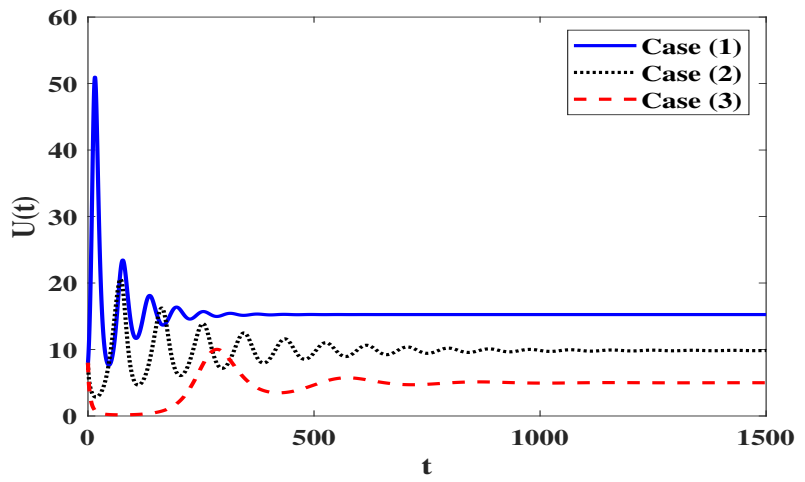

(b) Silent HIV-infected cells

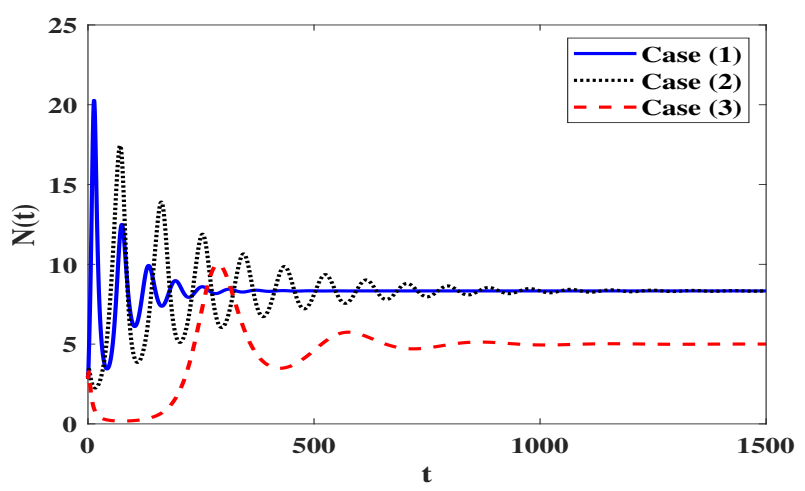

(d) Free HIV particles

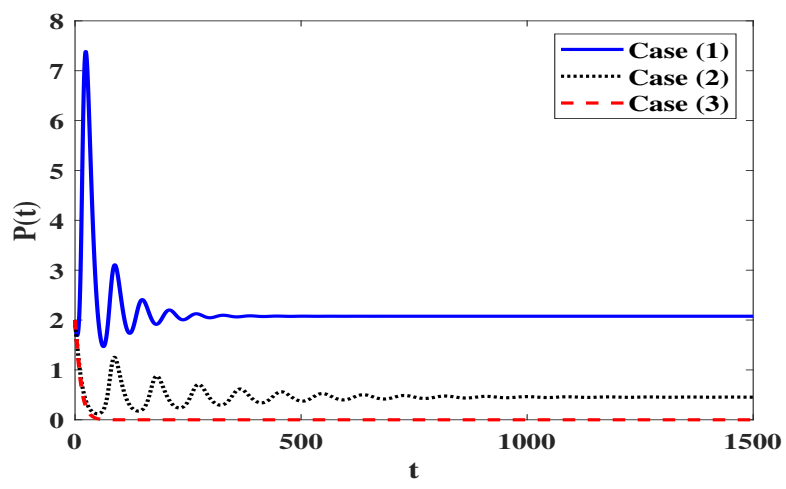

(e) HIV-specific CTLs

Figure 2: The evolution of HIV dynamics (2.1) under different modes of transmission. 


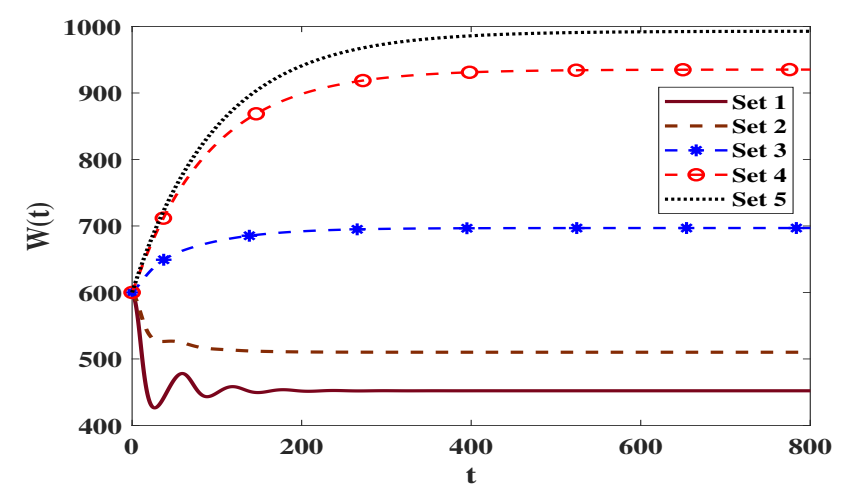

(a) Susceptible $\mathrm{CD} 4^{+} \mathrm{T}$ cells

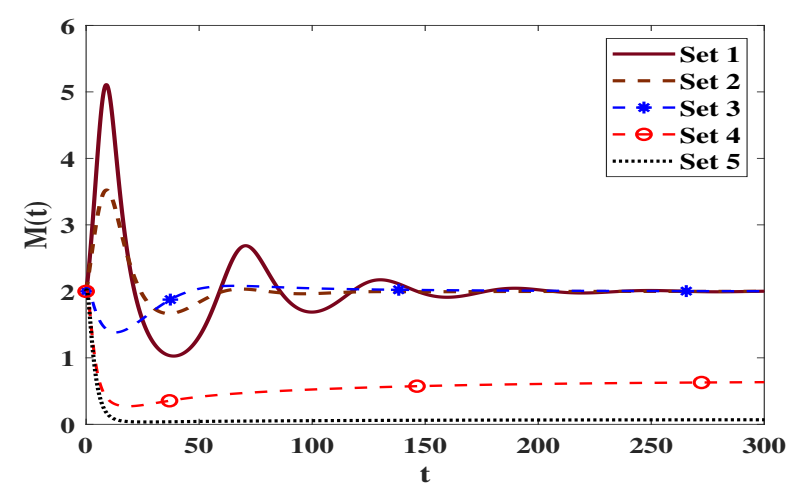

(c) Active HIV-infected cells

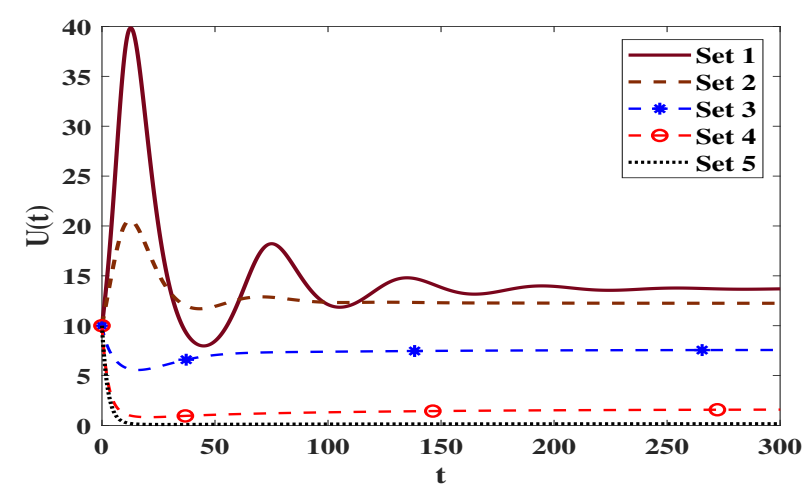

(b) Silent HIV-infected cells

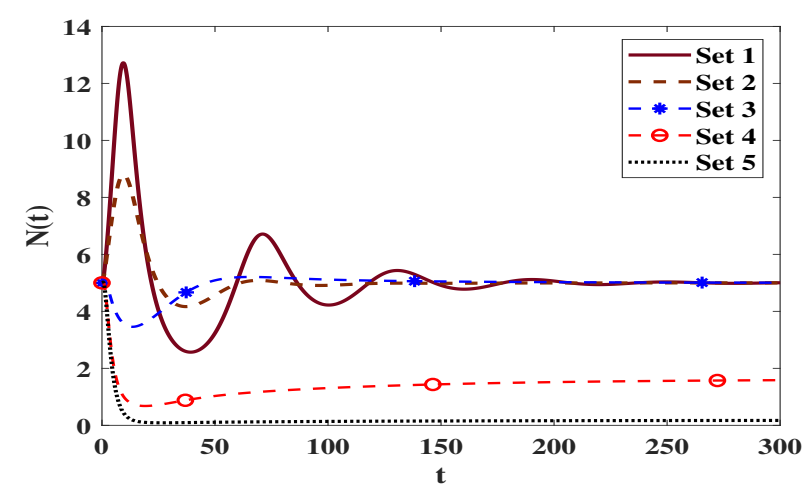

(d) Free HIV particles

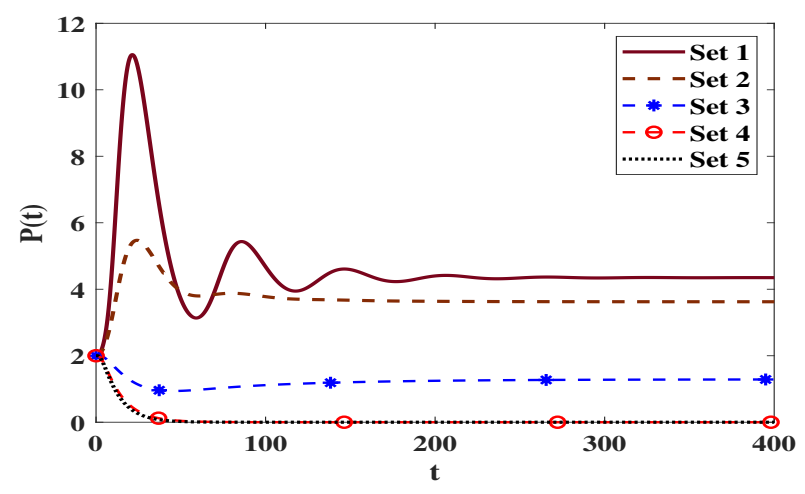

(e) HIV-specific CTLs

Figure 3: The influence of saturation parameters $\beta_{i}, i=1,2,3$ on the evolution of HIV dynamics (3.1).

\section{Conclusion}

In this paper, we investigated two HIV dynamics models with CTL-mediated immunity. The models involved the concentrations of susceptible CD4 ${ }^{+} \mathrm{T}$ cells, silent HIV-infected cells, active HIV-infected cells, free HIV particles and HIV-specific CTLs. We incorporated both VTC and CTC transmissions. The CTC infection is due to the contact between susceptible $\mathrm{CD} 4^{+} \mathrm{T}$ cells and silent or active HIV-infected cells. In the second model we considered a saturated incidence rate. For each model we showed that its solutions are nonnegative and bounded. We calculated the three equilibria of the model, the infection-free equilibrium, $\mathrm{\Xi}_{0}$, the chronic HIV infection equilibrium with inactive CTL-mediated immune response, $\bigoplus_{1}$, and the chronic HIV infection equilibrium with active CTL-mediated immune response, $\bigoplus_{2}$. We 
derived two threshold parameters, $\mathfrak{R}_{0}$ (the basic HIV reproduction number) and $\mathfrak{R}_{1}$ (the HIV specific CTL-mediated immunity reproduction number) which determine the existence and global stability of the three equilibria. By means of Lyapunov functions we proved the global asymptotic stability of the three equilibria. To illustrate the theoretical results, we performed some numerical simulations. We showed that the inclusion of CTC transmission decreases the concentration of susceptible CD4 ${ }^{+} \mathrm{T}$ cells and increases the concentrations of infected cells and free HIV particles.

Our proposed models (2.1) and (3.1) can be extended by incorporating age structure of the infected cells or diffusion $[3,4,10,33]$. Looking ahead to further developments an interesting perspective would be introducing a stochastic internal variable, as in [20], to account for virus mutations which generally develops by a stochastic dynamics. Moreover, since the exact analytical solutions of our proposed HIV dynamics models are not known, then we can only obtain approximate solutions of these models. Therefore, the discrete-time versions of the models need to be investigated (see, e.g., $[11,12,40])$. These extensions, indeed, require more investigations, therefore we leave it for future works.

\section{References}

[1] D. Adak, N. Bairagi, Analysis and computation of multi-pathways and multi-delays HIV-1 infection model, Appl. Math. Model., 54 (2018), 517-536. 1

[2] L. Agosto, M. Herring, W. Mothes, A. Henderson, HIV-1-infected CD4+ T cells facilitate latent infection of resting CD4+ T cells through cell-cell contact, Cell Rep., 24 (2018), 2088-2100. 1

[3] N. Bellomo, K. J. Painter, Y. Tao, M. Winkler, Occurrence vs. Absence of taxis-driven instabilities in a May-Nowak model for virus infection, SIAM J. Appl. Math., 79 (2019), 1990-2010. 5

[4] N. Bellomo, Y. Tao, Stabilization in a chemotaxis model for virus infection, Discrete. Cont. Dyn Syst.-S, 13 (2020), 105-117. 5

[5] H. Burger, A. L. Belman, R. Grimson, A. Kaell, K. Flaherty, J. Gulla, R. A. Gibbs, P. N. Nguyun, B. Weiser, Long HIV-1 incubation periods and dynamics of transmission within a family, Lancet, 336 (1990), 134-136. 1

[6] Á. G. Cervantes-Pérez, E. Ávila-Vales, Dynamical analysis of multipathways and multidelays of general virus dynamics model, Internat. J. Bifur. Chaos Appl. Sci. Engrg., 29 (2019), 30 pages. 1

[7] T.-W. Chun, L. Stuyver, S. B. Mizell, L. A. Ehler, J. A. M. Mican, M. Baseler, A. L. Lloyd, M. A. Nowak, A. S. Fauci, Presence of an inducible HIV-1 latent reservoir during highly active antiretroviral therapy, Proc. Natl. Acad. Sci. USA, 94 (1997), 13193-13197. 1

[8] R. V. Culshaw, S. Ruan, G. Webb, A mathematical model of cell-to-cell spread of HIV-1 that includes a time delay, J. Math. Biol., 46 (2003), 425-444. 1

[9] A. M. Elaiw, Global properties of a class of HIV models, Nonlinear Anal. Real World Appl., 11 (2010), 2253-2263. 2.3

[10] A. M. Elaiw, A. D. Al Agha, Analysis of a delayed and diffusive oncolytic M1 virotherapy model with immune response, Nonlinear Anal. Real World Appl., 55 (2020), 32 pages. 5

[11] A. M. Elaiw, M. A. Alshaikh, Stability of discrete-time HIV dynamics models with three categories of infected CD4 ${ }^{+}$ T-cells, Adv. Difference Equ., 2019 (2019), 24 pages. 5

[12] A. M. Elaiw, M. A. Alshaikh, Stability of a discrete-time general delayed viral model with antibody and cell-mediated immune responses, Adv. Difference Equ., 2020 (2020), 1-51. 5

[13] A. M. Elaiw, N. H. AlShamrani, Global stability of humoral immunity virus dynamics models with nonlinear infection rate and removal, Nonlinear Anal. Real World Appl., 26 (2015), 161-190. 2.3

[14] A. M. Elaiw, N. H. AlShamrani, Global stability of a delayed adaptive immunity viral infection with two routes of infection and multi-stages of infected cells, Commun. Nonlinear Sci. Numer. Simul., 86 (2020), 27 pages. 1

[15] A. M. Elaiw, N. H. AlShamrani, Stability of a general adaptive immunity virus dynamics model with multi-stages of infected cells and two routes of infection, Math. Methods Appl. Sci., 43 (2020), 1145-1175. 1

[16] A. M. Elaiw, S. F. Alshehaiween, Global stability of delay-distributed viral infection model with two modes of viral transmission and B-cell impairment, Math. Methods Appl. Sci., 43 (2020), 6677-6701. 1

[17] A. M. Elaiw, E. K. Elnahary, A. A. Raezah, Effect of cellular reservoirs and delays on the global dynamics of HIV, Adv. Difference Equ., 2018 (2018), 36 pages. 1

[18] A. M. Elaiw, I. A. Hassanien, S. A. Azoz, Global stability of HIV infection models with intracellular delays, J. Korean Math. Soc., 49 (2012), 779-794. 1

[19] A. M. Elaiw, A. A. Raezah, Stability of general virus dynamics models with both cellular and viral infections and delays, Math. Methods Appl. Sci., 40 (2017), 5863-5880. 1

[20] L. Gibelli, A. Elaiw, M. A. Alghamdi, A. M. Althiabi, Heterogeneous population dynamics of active particles: Progression, mutations, and selection dynamics, Math. Models Methods Appl. Sci., 27 (2017), 617-640. 5

[21] T. Guo, Z. Qiu, L. Rong, Analysis of an HIV model with immune responses and cell-to-cell transmission, Bull. Malays. Math. Sci. Soc., 43 (2020), 581-607. 1 
[22] J. K. Hale, S. M. Verduyn Lunel, Introduction to functional differential equations, Springer-Verlag, New York, (1993). 2.3

[23] G. Huang, Y. Takeuchi, W. Ma, Lyapunov functionals for delay differential equations model of viral infections, SIAM J. Appl. Math., 70 (2010), 2693-2708. 2.3

[24] D. Huang, X. Zhang, Y. Guo, H. Wang, Analysis of an HIV infection model with treatments and delayed immune response, Appl. Math. Model., 40 (2016), 3081-3089. 1

[25] S. Iwami, J. S. Takeuchi, S. Nakaoka, F. Mammano, F. Clavel, H. Inaba, T. Kobayashi, N. Misawa, K. Aihara, Y. Koyanagi, K. Sato, Cell-to-cell infection by HIV contributes over half of virus infection, Elife, 4 (2015), 18 pages. 1

[26] C. Jolly, Q. J. Sattentau, Retroviral spread by induction of virological synapses, Traffic, 5 (2004), 643-650.

[27] N. L. Komarova, D. Wodarz, Virus dynamics in the presence of synaptic transmission, Math. Biosci., 242 (2013), 161171. 1

[28] A. Korobeinikov, Global properties of basic virus dynamics models, Bull. Math. Biol., 66 (2004), 879-883. 2.3

[29] X. L. Lai, X. F. Zou, Modelling HIV-1 virus dynamics with both virus-to-cell infection and cell-to-cell transmission, SIAM J. Appl. Math., 74 (2014), 898-917. 1

[30] X. L. Lai, X. F. Zou, Modeling cell-to-cell spread of HIV-1 with logistic target cell growth, J. Math. Anal. Appl., 426 (2015), 563-584. 1

[31] X. Li, S. Fu, Global stability of a virus dynamics model with intracellular delay and CTL immune response, Math. Methods Appl. Sci., 38 (2015), 420-430. 1

[32] C. Lv, L. Huang, Z. Yuan, Global stability for an HIV-1 infection model with Beddington-DeAngelis incidence rate and CTL immune response, Commun. Nonlinear Sci. Numer. Simul., 19 (2014), 121-127. 1

[33] C. C. McCluskey, Delay versus age-of-infection-Global stability, Appl. Math. Comput., 217 (2010), 3046-3049. 5

[34] M. A. Nowak, C. R. M. Bangham, Population dynamics of immune responses to persistent viruses, Science, 272 (1996), 74-79. 1

[35] M. A. Nowak, R. M. May, Virus dynamics: Mathematical Principles of Immunology and Virology, Oxford University Press, Oxford, (2000). 1

[36] H. Sato, J. Orenstein, D. Dimitrov, M. Martin, Cell-to-cell spread of HIV-1 occurs within minutes and may not involve the participation of virus particles, Virology, 186 (1992), 712-724. 1

[37] X. Y. Shi, X. Y. Zhou, X. Y. Song, Dynamical behavior of a delay virus dynamics model with CTL immune response, Nonlinear Anal. Real World Appl., 11 (2010), 1795-1809. 1

[38] H. Y. Shu, L. Wang, J. Watmough, Global stability of a nonlinear viral infection model with infinitely distributed intracellular delays and CTL immune responses, SIAM J. Appl. Math., 73 (2013), 1280-1302. 1

[39] A. Sigal, J. T. Kim, A. B. Balazs, E. Dekel, A. Mayo, R. Milo, D. Baltimore, Cell-to-cell spread of HIV permits ongoing replication despite antiretroviral therapy, Nature, 477 (2011), 95-98. 1

[40] H. Sun, J. Wang, Dynamics of a diffusive virus model with general incidence function, cell-to-cell transmission and time delay, Comput. Math. Appl., 77 (2019), 284-301. 5

[41] X. Tian, R. Xu, Global stability and Hopf bifurcation of an HIV-1 infection model with saturation incidence and delayed CTL immune response, Appl. Math. Comput., 237 (2014), 146-154. 1

[42] J. Wang, M. Guo, X. Liu, Z. Zhao, Threshold dynamics of HIV-1 virus model with cell-to-cell transmission, cell-mediated immune responses and distributed delay, Appl. Math. Comput., 291 (2016), 149-161. 1

[43] J. Wang, C. Qin, Y. Chen, X. Wang, Hopf bifurcation in a CTL-inclusive HIV-1 infection model with two time delays, Math. Biosci. Eng., 16 (2019), 2587-2612. 1

[44] W. Wang, X. Wang, K. Guo, W. Ma, Global analysis of a diffusive viral model with cell-to-cell infection and incubation period, Math. Methods Appl. Sci., 43 (2020), 5963-5978. 1

[45] J. K. Wong, M. Hezareh, H. F. Gunthard, D. V. Havlir, C. C. Ignacio, C. A. Spina, D. D. Richman, Recovery of replication-competent HIV despite prolonged suppression of plasma viremia, Science, 278 (1997), 1291-1295. 1

[46] Y. Yang, L. Zou, S. G. Ruan, Global dynamics of a delayed within-host viral infection model with both virus-to-cell and cell-to-cell transmissions, Math. Biosci., 270 (2015), 183-191. 1

[47] H. Zhu, Y. Luo, M. Chen, Stability and Hopf bifurcation of a HIV infection model with CTL-response delay, Comput. Math. Appl., 62 (2011), 3091-3102. 1 